\title{
APDM: An Adaptive Multi-Priority Distributed Multichannel MAC Protocol for Vehicular Ad Hoc Networks in Unsaturated Conditions
}

\author{
Caixia Song, ${ }^{\mathrm{a}, \mathrm{w}}$, Guozhen Tan ${ }^{\mathrm{a}}$, Chao $\mathrm{Yu}^{\mathrm{a}}$, Nan Ding ${ }^{\mathrm{a}}$, Fuxin Zhang ${ }^{\mathrm{a}}$ \\ ${ }^{a}$ College of Computer Science and Technology, Dalian University of Technology, Dalian 116024, \\ China; \\ ${ }^{b}$ College of Science and Information, Qingdao Agricultural University, Qingdao 266109, China.
}

\begin{abstract}
Vehicular ad hoc networks (VANETs) employ multichannel to provide a variety of safety and non-safety applications. Safety applications require reliable and timely transmission, while non-safety applications need high network throughput. IEEE 802.11p and IEEE 1609.4 protocol divide the bandwidth into seven channels. One control channel $(\mathrm{CCH})$ is to serve safety applications and the other six service channels (SCHs) to serve non-safety applications. The IEEE 1609.4 protocol specifies an alternating scheme to allow vehicles to switch between two types of applications. However, the IEEE 1609.4 multichannel media access control (MAC) protocol has limitations on its capability of supporting either delay- or throughput-sensitive applications. In this paper, we propose an adaptive multi-priority distributed multichannel (APDM) MAC protocol for VANETs. Considering that in realistic VANETs, the queue of MAC layer is far from saturated. We assume that generated packets with different priorities arrive at the MAC layer in a Poisson manner. A Markov analytical model is conducted to optimize the packet transmission probabilities and adjust the ratio between $\mathrm{CCH}$ interval and $\mathrm{SCH}$ interval dynamically according to the real-time traffic in a distributed way. An $\mathrm{M} / \mathrm{M} / 1$ queue model is then adopted to analyze the time performance. Extensive simulation results show that the APDM MAC protocol can ensure prioritized transmission of safety packets, reduce the transmission delay of packets and enhance the unsaturated and saturated throughput of SCHs.
\end{abstract}

\footnotetext{
*Corresponding author

Email address: cassiesong@mail.dlut.edu.cn(Caixia Song)()
}

Preprint submitted to Elsevier

July 11, 2016

(C) 2016. This manuscript version is made available under the Elsevier user license http://www.elsevier.com/open-access/userlicense/1.0/ 
Keywords: Vehicular Ad Hoc Networks (VANETs), IEEE 1609.4, Medium Access Control (MAC), Unsaturated Traffic, Markov Model.

\section{Introduction}

Vehicular ad hoc networks (VANETs) utilize on-board unit (OBU) and road side unit (RSU) to achieve real-time vehicle-to-vehicle (V2V) and vehicle-to-infrastructure (V2I) communications [1]. Vehicles rely on V2V and V2I to support various publicsafety applications (e.g., emergency brake, lane change warning, cooperation collision avoidance) as well as non-safety applications (e.g., digital map and media downloading, Internet services) [1][2][3].

However, development and operation of VANETs have various obstacles due to high node mobility and constantly varying vehicle density. The main dilemma in VANETs is that safety messages with high priority require reliable and timely data transmission, while non-safety transmissions such as using Google map and other infotainment services need heavy throughput of the network.

In the $5.9 \mathrm{GHz}$ band, a $75 \mathrm{MHz}$ bandwidth is to be used exclusively for vehicular communications. IEEE $802.11 \mathrm{p}$ [4] is specially used for wireless access in vehicular environments (WAVE). And it is based on enhanced distributed channel access (EDCA) with priority. Above the IEEE 802.11p protocol, the IEEE 1609.4 specifies multichannel architecture [4]. The channel is divided into two different types according to the categories of transmitted messages: a shared control channel $(\mathrm{CCH})$ for safety-related messages transmission (e.g., emergency messages, beacon messages and other control messages like WAVE Service Advertisement (WSA))[5], and six service channels (SCHs) for non-safety (e.g, comfort and entertainment) data transmission. The channel time is divided into synchronization intervals with a fixed length of 100 ms. A synchronization interval (SI) includes a 50ms $\mathrm{CCH}$ interval (CCHI) and a 50ms SCH interval (SCHI), as shown in Fig. 1. During the CCHI, all devices need tune to $\mathrm{CCH}$ for the transmission of safety-related messages or WSA messages, while in the SCHI, devices can optionally tune to the specific SCH to deliver non-safety messages. In IEEE 1609.4 protocol, a coordinated universal time (UTC) is used for time synchronization, which obtained from global positioning system (GPS) [4]. For single-radio devices, synchronous and periodic switching between $\mathrm{CCH}$ and $\mathrm{SCHs}$ is mandatory.

However, the mandatory channel switching can cause safety data expired to drop. For example, if the residual time before the end of CCHI is shorter than the transmission delay of a safety packet, it cannot be disseminated. Thus, such a safety packet can be dropped when its lifetime is exceeded [6]. On the other hand, the $50 \mathrm{~ms} \mathrm{SCH}$ 


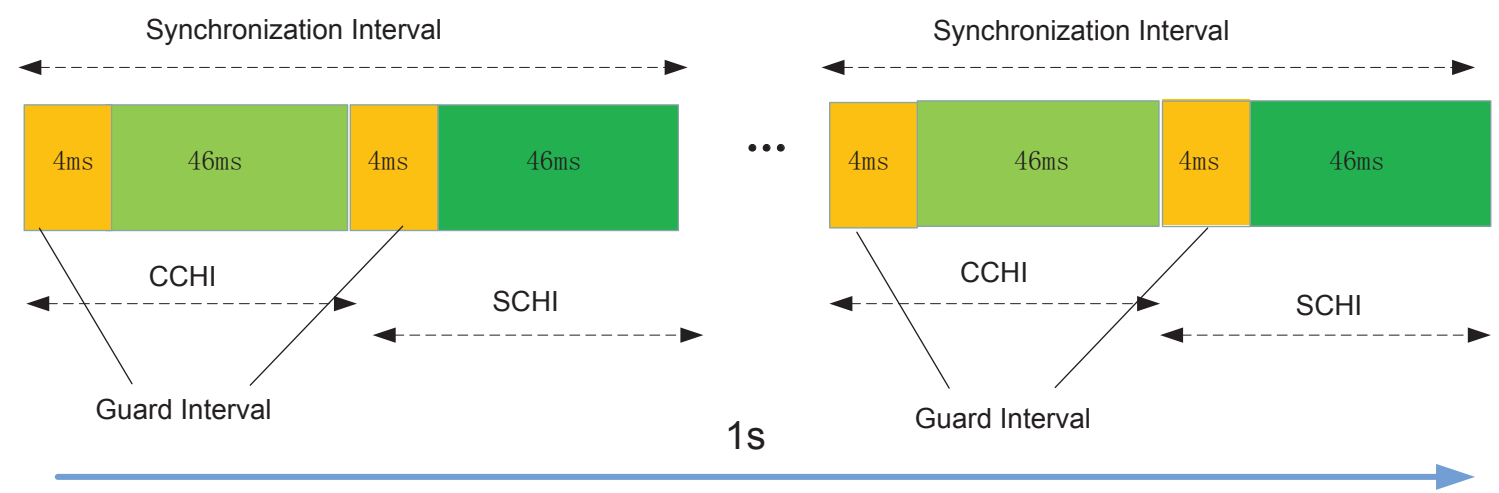

Figure 1: IEEE 1609.4 multichannel alternating operation

interval is not enough to transmit bulk non-safety packets. Therefore, due to the issue of safety packet expiry and non-safety packet starvation [7], the fixed interval does not fit for the real environment of the VANETs and cannot satisfy either delayor throughput-sensitive applications [7][8][9][10][11]. Under congested traffic environments, the limited $50 \mathrm{~ms}$ of CCHI cannot guarantee reliable and timely delivery of safety-related messages. On the contrary, when the vehicle density is light but heavy network service is required, the $\mathrm{CCH}$ only needs a little time to transmit a small amount of safety-related messages, while SCHs cannot provide enough time to perform some a large amount of bandwidth consuming applications, such as digital map update and video download. In order to guarantee prioritized transmission of safety-related messages and enhance the throughput of SCHs, a great deal of work [5][9][10][12] has been done in recent years to address this issue. Most of the work, however, is focused on saturated network conditions, which means that nodes always have packets to deliver. Provided that every node is indeed under saturation, the resulting model is remarkably accurate. However, under saturation condition, traffic characteristics in VANETs, such as inter arrival time and burstiness are not taken into account. In VANETs, on $\mathrm{CCH}$, beacon messages (with a packet arrival rate of $10 \mathrm{~Hz}$ [13][14]) for neighboring awareness is the majority of the network traffic. Although, there are emergency messages and WSA messages, they happen only occasionally [15] and are typically bursty in nature. In addition, on $\mathrm{CCH}$, the delay requirements of broadcast packets and due to lifetime expiration, they are usually dropped at the end of the CCHI. Therefore, the queues are far from saturated on $\mathrm{CCH}[6][14][16]$. For example, for each disseminated beacon packet on $\mathrm{CCH}$, we suppose that a packet' size is 500 bytes containing 300 bytes of payload and 200 bytes for security consideration. We choose transmission rate of $6 \mathrm{Mbps}$, the duration for successful transmitting this packet is $(500 * 8) /(6 * 1000) \approx 0.7$ ms. Thus, without 
considering competition between nodes, only the interval of packet arrival is approximately $0.7 \mathrm{~ms}$, the queue is ensured in saturated conditions. In general, arrival rate with 1000 packets/s for different ACs on $\mathrm{CCH}$, each queue is guaranteed with saturated traffic load [17][18]. In communication-enabled vehicular safety applications, the transmission rate between $1 \mathrm{~Hz}$ and $50 \mathrm{~Hz}$ on $\mathrm{CCH}$ is required [19]. In fact, in a high vehicle density, a reduction in basic safety message transmission rate does not necessarily compromise the vehicles safety. Since drivers become more alert in these conditions and tend to lower their speeds, thus, the probability of accidents drops [20][21]. On the other hand, it is quite common that a node's idle time is more than its transmission duration, and the packet arrival rate is variable and differs with different packet priorities [6][16]. Therefore, the unsaturated conditions are more in line with the characteristics of the VANETs.

In this paper, we propose an adaptive multi-priority distributed multichannel (APDM) MAC protocol in unsaturated conditions. According to different priorities of packets in VANETs, the APDM MAC protocol using Markov chain analysis to adjust the ratio between $\mathrm{CCHI}$ and SCHI in a distributed way, so as to first guarantee reliable and timely transmission of safety messages, and then achieve maximal throughput of non-safety applications. The delay of a safety packet or a service packet is derived by using $\mathrm{M} / \mathrm{M} / 1$ queue theory. The main contributions of this paper are threefold:

1) To guarantee preemptive transmission of safety critical packets, the APDM $\mathrm{MAC}$ protocol sorts packets on $\mathrm{CCH}$ into two priority categories based on the degree of emergency. We classify safety-related packets (e.g., emergency brake, collision risk warning, emergency vehicle warning, periodic beacon) as the higher priority access category $\left(A C_{1}\right)$, and other control packets like WSA packets as the lower priority $\left(A C_{2}\right)$. Packets differentiation mechanism is proposed to guarantee the prioritized transmission of the safety-related packets.

2) To characterize the feature of real environment in VANETs, we present a Poisson process and a Markov chain model to optimize the interval ratio between the CCHI and the SCHI to satisfy dynamic traffic flow under both unsaturated and saturated conditions. More $\mathrm{CCH}$ interval is allocated to ensure reliable and timely transmission of safety packets under dense traffic flow, and on the contrary, more SCH interval is allocated to improve the system throughput and the channel utilization in light traffic flow with heavy non-safety applications.

3) The number of vehicle nodes is derived by a simple but accurate method in a distributed manner, and the APDM MAC protocol can work with or without RSUs.

The rest of this paper is organized as follows: Section 2 reviews related work. The proposed APDM MAC protocol is described in detail in Section 3. Section 4 
first presents the Markov chain models to analyze the transmission probabilities for different kinds of packets, so as to drive an optimal ratio between CCHI and SCHI, and then conducts a performance analysis. Simulation evaluation is given in Section 5. Finally, Section 6 concludes this paper.

\section{Related Works}

In order to take advantage of channel resources in VANETs, an increasing number of researchers have studied the multichannel MAC protocol. IEEE 1609.4 protocol specified four multichannel MAC protocols based on channel assignment and coordination principles: Continuous, Alternating, Immediate, and Extended Channel Access [4]. However, only the alternating channel access scheme has been shown to be the optimal solution to support both safety and non-safety applications [4][5], and most researchers in VANETs have focused on this scheme in their research (also called Split Phase) [6][8][9][10][11][22] [23][24]. The alternating channel access scheme is the most successful one because that at a time, it can operate on only one channel, and thus suitable for single radio transceivers. Therefore, we focus on this scheme for the single-radio transceivers in this paper.

VANETs have various kinds of applications including traffic safety, traffic efficiency (such as speed management, co-operative navigation) and infotainment. Safetyrelated applications require efficient and reliable transmission performance. Due to the large of number of nodes within a confined area and vehicles' high mobility, the literatures [6][25][26] indicated that vehicular networks suffer from low reliability due to high transmission collision especially in situations of high vehicle density. This is because when the vehicles synchronize at the start of $\mathrm{CCH}$ interval, it is very likely to occur that the frames collide with each other, which is called synchronized frame collisions. Moreover, due to life expiration, the safety packets are normally dropped at the end of CCHI.

To overcome this serious problem, reseachers proposed cluster based MAC protocol [27][28] or time division multiple access (TDMA) [22][29]. They assign deterministic time slots for vehicles or select cluster header to transmit safety-related messages. However, in order to minimize or avoid transmission collisions, how long the $\mathrm{CCH}$ interval should be is the primary question that must be answered.

The authors in [6][7][9][10][24] studied the above question using model analysis and simulation analysis. The authors analyzed the existing safety and non-safety applications in various vehicular networks conditions and realized that the fixed CCHI and SCHI (50ms each other) could not ensure effective communication under time-varying vehicular environments. For example, under congested vehicular traffic 
conditions, the limited 50ms CCHI cannot guarantee timely and reliable delivery of safety-related packets. On the other hand, they also realized that in a light network environment, the SCHI of $50 \mathrm{~ms}$ could not provide enough time to perform some massive bandwidth consuming applications, thus the bandwidth is wasted. Therefore, to improve the communication performance, according to the network traffic situation, dynamically adjusting the ratio of CCHI and SCHI is necessary.

A variable CCH interval (VCI) multichannel protocol is presented in [9]. The VCI scheme divides CCHI into a safety interval, a WSA interval, and dynamically adjusts the ratio between $\mathrm{CCH}$ interval and $\mathrm{SCH}$ interval based on the traffic conditions. It can guarantee reliable transmissions of safety messages to improve the saturation throughput of SCHs. In a high-dense VANETs environment and an emergent situation, many vehicles contend for $\mathrm{CCH}$ simultaneously to send safety-related packets and exchange control packets (e.g., WSA) on $\mathrm{CCH}$, which incur intense channel contention and long delay for safety packet transmission [6]. It is necessary to classify the packets on $\mathrm{CCH}$ with different priorities to guarantee priority transmission of safety packets. In [30], Khabazian et al. proposed that the traffic generated by event-driven safety applications has higher priority than the other network traffic. The paper showed that the average number of nodes receiving high-priority messages is a function of the dissemination range. However, the paper assumed that only one event-driven safety packet can be transmitted in the network at any time, nevertheless, several safety-related packets can be propagated in VANETs at the same time. The works in [10] differentiates the packets into multiple priorities on the $\mathrm{CCH}$ according to the time slotted p-persistent channel access mechanism. It can obtain optimal CCHI and SCHI based on the maximum saturated throughput. However, this scheme only takes saturation conditions into consideration, while in VANETs, packets' queue in MAC layer is far from saturation conditions [6][16]. Moreover, in [9][10], the works assume that RSU knows the number of nodes, which may be impossible in distributed scenarios. Different from the above methods, our proposed APDM MAC protocol can dynamically adjust the interval ratio between CCHI and SCHI by considering multi-priority packets in a distributed way under unsaturated conditions.

\section{APDM: An Adaptive Multi-Priority Distributed Multichannel MAC Protocol}

In this paper, to ensure quality of services (QoS) for different traffic flow, we propose an adaptive multi-priority distributed multichannel (APDM) MAC protocol. The APDM MAC protocol estimates the number of neighbouring vehicles in a 
distributed manner, and then uses Markov chain and M/M/1 queue model to derive the transmission probabilities of packets of different priorities. According to derived the transmission probabilities in unsaturated conditions, the optimal ratio between CCHI and SCHI can be achieved, so as to firstly guarantee the preemptive priority transmission of the safety packets, and then obtain maximal service packets throughput on SCHs.

The APDM MAC protocol uses UTC [4] mechanism for time synchronization among all vehicles by GPS. According to the traffic condition, a SI is divided into the adjustable CCHI and SCHI. During the CCHI, nodes not only disseminate the safety-related messages, but also conduct statistics and measurement for channel coordination and channel assignment. As shown in Fig. 2, the CCHI is further departed into multi-priority broadcast interval and random contention interval. A new $\mathrm{CCH}$ interval starts at the multi-priority broadcast interval, during which the vehicle with the minimal MAC address (MAC_ID), called optimizing node (ON), broadcasts the optimal duration of the CCHI. The optimal interval of the CCHI is in a special packet called optimal ratio packet (ORP). Based on the receiving ORP, vehicles adjust the random contention interval. During the random contention interval, nodes disseminate the safety messages, and make SCHs reservation for WSA packets. Nodes will tune to the reserved SCHs to conduct service packet transmissions at the end of the random contention interval.

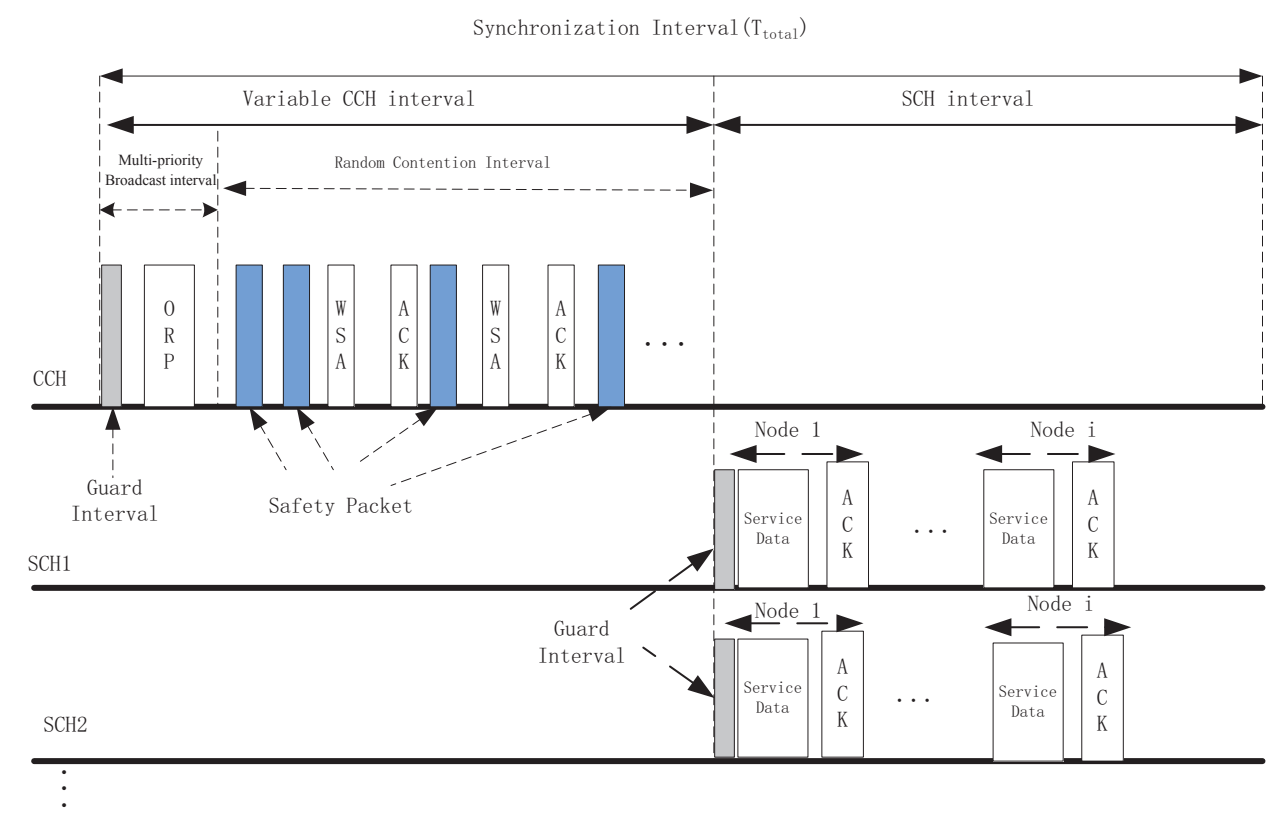

Figure 2: The frame of APDM MAC protocol 


\subsection{Multi-Priority Transmission Strategy}

In order to guarantee preemptive priority reliable transmission of safety-related packets in unsaturated conditions, APDM MAC protocol classifies the packets on the $\mathrm{CCH}$ into two priorities. The event-driven safety packets and periodic beacons are classified as safety-related packets with higher priority $\left(A C_{1}\right)$, while the control packets like WSA packets are classified as non-safety packets with lower priority $\left(A C_{2}\right)$. Classifying the different priorities can increase the delivery of safety-related packets on $\mathrm{CCH}$ under dense conditions. By utilizing Markov chain models, we derive the transmission probability of $\tau_{e}$ and $\tau_{s}$ in an arbitrary slot time [31], where $\tau_{e}$ and $\tau_{s}$ are the transmission probabilities of the packets with $A C_{1}$ and $A C_{2}$, respectively. Therefore, according to the arrival rate of generated packets and the number of nodes, the optimal interval ratio of the CCHI and SCHI can be derived. More detailed derivation of the $\tau_{e}$ and $\tau_{s}$ will be presented in Section 4.2.

APDM nodes adopt EDCA of IEEE 802.11e to support QoS for different applications. For each $A C_{i}(i=1,2)$, the transmission opportunities are affected by arbitration inter-frame space number (AIFSN) and contention window (CW). Based on specification of IEEE 802.11e, the backoff timer will decrease after an idle time slot plus AIFSN $\left(A C_{i}\right)$ time slots. For improving the average system throughput and timely dissemination of safety packets, we apply small AIFSN value with AIF$\operatorname{SN}\left(A C_{1}\right)=2$ and $\operatorname{AIFSN}\left(A C_{2}\right)=3$ [32]. Due to the smaller AIFSN of $A C_{1}$, the transmission probability of a packet with $A C_{1}$ is greater than that of a packet with $A C_{2}$, i.e., $\tau_{e}>\tau_{s}$. At the beginning of any time slot, when an APDM node senses the channel is idle and its backoff timer is zero, then it attempts to transmit data. The backoff timer is frozen when the channel is busy. The multi-priority scheme can decrease nodes' collision and make preemptive priority access to $\mathrm{CCH}$ of safety packets.

\subsection{Variable Interval Ratio Between the $C C H$ Interval and SCH Interval}

In the IEEE 1609.4 standards [4], proper bandwidth to satisfy transmission of both safety and non-safety application stream is not provided by fixed CCHI and SCHI. In heavy traffic flow and higher arriving rate of safety packets, the shared $\mathrm{CCH}$ is contended to access by a large number of safety-related packets. The CCHI need to be adjusted more than $50 \mathrm{~ms}$ to guarantee the timely and reliable transmission of safety-related packets. On the contrary, when the traffic is sparse but heavy service transmission is required, the SCHI should be extended to improve the aggregated throughput [9][10] .

According to the APDM MAC protocol, the nodes detect the channel condition and calculate the number of the neighbouring vehicles according to packets received 
from the neighbouring vehicles. The nodes count the number of the low and high priority packets, then calculate the busy or idle probability of the channel. Based on the information, nodes use the Markov chain and M/M/1 queue model to derive the optimal CCHI which is included in ORP. As illustrated in Fig. 2, the ON broadcasts an ORP packet to its neighbouring vehicles at the beginning of CCHI of the next SI. The ORP packets will be broadcasted twice to guarantee reliable transmission. In congested conditions, the ORP packet may not be received by some of the neighbouring vehicles. To handle this question, a field about the latest duration of CCHI is added into WSA packets. The nodes sending WSA packets will fill this field with latest interval of CCHI, while other nodes will use zero to fill this field. Moreover, in the APDM MAC protocol, firstly, the length of CCHI is ensured to disseminate all the safety-related packets. Secondly, the optimal length of CCHI should be in such a state that the number of successful reserved WSA packets on $\mathrm{CCH}$ equals the number of packets transmitted on all SCHs [9]. In section 4.4, we will give the optimal interval by the theoretical analysis. For each vehicle, the initial duration of CCHI is $50 \mathrm{~ms}$ and according to the network traffic condition in the upcoming SI, this value is dynamically adjusted.

\subsection{SCH Selection and Access Reservation Scheme}

The IEEE1609.4 is a contention-based MAC access mechanism. In order to improve the throughput of service packets on $\mathrm{SCHs}$, it is necessary to adopt a new multichannel coordination and assignment mechanism to reduce collisions on SCHs [9][10]. By channel reservation on $\mathrm{CCH}$, multichannel coordination mechanism provides contention-free SCHs. While multichannel assignment is how to select idle SCHs for nodes.

Each APDM node maintains a neighboring information list (NIL) and a channel usage list (CUL), as shown in Table 1. The NIL (Table 1(a)) stores neighbouring nodes' MAC_ID, time to receive the neighbouring nodes' message, as well as SCH and TxSlot used by the neighbouring nodes, where the TxSlot represents the slot time of the node transmission message. The CUL (Table 1(b)) stores the available TxSlots on each SCH. During the random contention interval, each service provider broadcasts a WSA packet with transmission probability $\tau_{s}$ to reserve SCHs. The operation of the SCHs access reservation and selection procedure is as follows:

1) When an APDM node has service messages to deliver, it will send a WSA. The WSA packet contains the service provider's MAC address, service information, its CUL and the other information [4].

2) When the receiver receives the WSA and is interested in the service, it will select the common SCH and TxSlot based on the CULs of both the sender and the 
Table 1: TWO LISTS IN APDM MAC PROTOCOL

(a) Node's NIL

\begin{tabular}{|c|c|c|c|}
\hline $\begin{array}{c}\text { Node } \\
\text { MAC_ID }\end{array}$ & $\begin{array}{c}\text { Receiving } \\
\text { Time }\end{array}$ & SCH & TxSlot \\
\hline ID1 & Time1 & 3 & 2 \\
ID2 & Time2 & 3 & 3 \\
ID3 & Time3 & 2 & 4 \\
$\ldots$ & $\ldots$ & $\ldots$ & $\ldots$ \\
\hline
\end{tabular}

(b) Node's CUL

\begin{tabular}{|c|c|}
\hline SCH & $\begin{array}{c}\text { Available } \\
\text { Slots }\end{array}$ \\
\hline 1 & 2 \\
2 & 2,3 \\
3 & 4 \\
4 & 3 \\
\hline
\end{tabular}

receiver. And then the receiver will send an ACK including the selected [SCH,TxSlot] to the sender.

3) Both the sender and the receiver switch to the selected $\mathrm{SCH}$ in the selected TxSlot to transmit packets.

4) Neighbouring nodes overhearing the ACK messages, update their CULs and NILs.

In a sparse traffic, if only s few service providers have service packets to transmit on SCHs, there are many idle SCHs and available TxSlots. To improve the throughput, a service provider may transmit multiple packets in a SCHI. However, in a dense vehicle environment, since too many service providers want to deliver their service packets, to be fair, each provider transmits only one packet in a SCHI. The SCH reservation scheme can provide contention-free transmission on SCHs and thus avoid invalid transmission of service information without any subscriber. Accordingly, the throughout of the network can be greatly maximized.

\subsection{Estimating Number of Nodes in a Distributed Way}

Every vehicle has unique MAC address and can determine unique MAC_ID. Each APDM node sends the packet including the MAC_ID and the receiver will recognize different nodes by MAC_ID. In order to avoid duplication of statistics, a checking duplication method is proposed. If a vehicle receives a packet and reads MAC_ID from the packet, it then checks its NIL to add a new record or replace the old record with the new one.

On the other hand, an APDM node divides the time into periodic observation interval (OI). Due to a node leaving the network without any notification information, for avoiding the NIL too large, every few OIs, each node should update its NIL and remove expired nodes' records. Considering that long time will bring about a lot of expired nodes, while short time will cause frequent updates, we set the OI be 1s.

From the NIL, an APDM node can calculate the number $N$ of neighbouring vehicles. With $N$, nodes use the Markov chain model based on Poisson arrival rate 
to compute the transmission probabilities $\tau_{e}$ and $\tau_{s}$, and then derive the optimal CCHI. The optimal CCHI is included in an ORP, and will be sent by ON. A vehicle compares its own MAC_ID with the MAC_ID in NIL to check whether its MAC_ID is the minimal value. Once a vehicle's MAC_ID is the minimal value, then at the beginning of the following SI, it broadcasts the ORP twice. Note that if there is a RSU, it can act as ON by setting the RSU of MAC_ID a very small value (zero) to make sure it is the smallest value in the communication range. Estimating the number of nodes and broadcasting the ORP are conducted in a distributed way. Thus, it can work well in scenarios with or without RSUs.

\section{MODEL ANALYSIS}

In the APDM MAC protocol, according to the general traffic conditions, the optimal interval ratio between CCHI and SCHI can be dynamically adjusted, and the protocol satisfies both delay-sensitive and throughput-sensitive applications. To derive the optimal interval, we first employ Markov chain to derive the stationary probability that an APDM node transmits a safety packet or a WSA packet in each time slot. Then, on the basis of carrier sense multiple access/collision avoidance (CSMA/CA), we calculate the average time of a safety packet transmission or a WSA reservation, respectively. Finally, the optimal length of CCHI will be achieved. Moreover, the delay of a safety packet or a service packet will be derived by using M/M/1 queue theory. For convenience, Table 2 summarizes the main parameters together with their significances in the model analysis. 
Table 2: MAIN PARAMETERS FOR MODEL ANALYSIS

\begin{tabular}{|c|c|}
\hline Parameter & Description \\
\hline$N$ & The number of nodes. \\
\hline$\tau_{i}$ & The transmission probability of the type of $i$ packet at each time slot. \\
\hline$P_{i, s u c}$ & The probability of the type of $i$ packet is successfully transmitted. \\
\hline$P_{e, c o l}$ & The collision probability of transmission a safety packet caused by only safety packets. \\
\hline$P, c o l$ & The collision probability of transmission a WSA packet caused by only WSA packets. \\
\hline$P_{e s, c o l}$ & The collision probability of transmission a packet caused by safety packets and WSA packets. \\
\hline$P_{e}$ & The collision probability of transmission a safety packet. \\
\hline$P_{s}$ & The collision probability of transmission a WSA packet. \\
\hline$P_{i, e m p}$ & The probability that the type of $i$ queue is empty. \\
\hline$P_{b}$ & The probability that the channel is busy. \\
\hline$I_{i, e m p}$ & The state with an empty queue belongs to the type of $i$ packet \\
\hline$\beta$ & The ratio of CCHI and SCHI. \\
\hline$\sigma$ & The duration of an empty slot. \\
\hline$\delta$ & The duration of the propagation delay. \\
\hline$D I F S$ & A distributed inter-frame space (DIFS) time. \\
\hline$S I F S$ & A short inter-frame space(SIFS) time. \\
\hline$S A F E$ & The duration for transmitting the payload of a safety packet. \\
\hline$W S A$ & The duration for transmitting the payload of a WSA packet. \\
\hline$A C K$ & The duration for transmitting the payload of an ACK packet. \\
\hline$T_{e, s u c}$ & The duration for successful transmitting a safety packet. \\
\hline$T_{s, s u c}$ & The duration for successful transmitting a WSA packet. \\
\hline$T_{e, c o l}$ & The duration for a transmission collision caused by safety traffic. \\
\hline$T_{s, \mathrm{col}}$ & The duration for a transmission collision caused by WSA traffic. \\
\hline$\overline{T_{E S}}$ & $\begin{array}{l}\text { The expected time for transmitting a packet which it is considering } \\
\text { a state of successful transmission, a collision or the channel being idle. }\end{array}$ \\
\hline$X_{e}$ & $\begin{array}{l}\text { The duration from the time instant when a safety packet becomes } \\
\text { the head of the MAC queue to the time instant that it is successfully transmitted. }\end{array}$ \\
\hline$X_{s}$ & $\begin{array}{l}\text { The duration from the time instant when a WSA packet becomes } \\
\text { the head of the MAC queue to the time instant that it successfully makes reservation. }\end{array}$ \\
\hline$T_{c c h}$ & The time duration of the variable CCHI. \\
\hline$T_{s c h}$ & The time duration of the variable SCHI. \\
\hline$T_{c c h i}$ & The time duration of the fixed CCHI(50ms). \\
\hline$T_{\text {schi }}$ & The time duration of the fixed SCHI(50ms). \\
\hline$T_{\text {safe }}$ & The time duration for transmitting a safety packet. \\
\hline$T_{w s a}$ & The time duration for transmitting a WSA packet. \\
\hline$T_{a c k}$ & The time duration for transmitting an ACK packet. \\
\hline$T_{\text {safe }}^{\prime}$ & The total time duration for transmitting all safety packets. \\
\hline$T_{w s a}^{\prime}$ & The total time duration for WSA packets reservation. \\
\hline$T_{\text {total }}$ & The time duration of a synchronization interval. \\
\hline$W_{e}$ & The CW size for safety packets. \\
\hline$W_{s, i}$ & The CW size for WSA packets in the ith backoff stage. \\
\hline$N_{s c h}$ & The number of available SCHs in VANETs. \\
\hline$S_{s c h}$ & The throughput of the system on SCHs. \\
\hline
\end{tabular}

Note: $\mathrm{i}$ in the parameters except $T_{c c h i}, T_{s c h}$ and $W_{s, i}$ stands for e and $\mathrm{s}$ which means safety and WSA, respectively. 


\subsection{Multi-Priority Transmission Model}

There are $N$ APDM nodes in our analytical model to deliver safety packets and WSA packets in the network. Based on the backoff process in EDCA, we present two Markov chain models to obtain the stationary probability $\tau_{e}$ and $\tau_{s}$ under unsaturation conditions and saturated conditions. The model adopts the following assumptions: (a) the channel conditions are ideal(i.e., no exposed terminals and hidden terminals). (b) the collision probability is independent and constant for each vehicle, irrespective of the number of retransmissions already experienced [31].

Let $b_{e}(t)$ denote a stochastic process representing the backoff timer value of safety traffic at time $t$. Since the transmission method of safety packets is broadcast without backoff stages, so the fixed contention window $(\mathrm{CW})$ is denoted by $W_{e}$. The state of safety data is described by $\left\{k ; k \in\left[0, W_{e}-1\right]\right\}$. $I_{e, i d l e}$ stands for an idle state of an APDM node with an empty safety queue. The stationary distribution of the idle state and the backoff state $k$ are denoted by $b_{I_{e}}$ and $b_{e, k}$, respectively. A onedimensional Markov chain is illustrated in Fig. 3. At each time slot, the backoff timer subtracts one and the one-step transition probabilities are:

$$
\begin{cases}P\{k \mid k+1\}=1-P_{b} \quad 0 \leq k \leq W_{e}-2 \\ P\{k \mid 0\}=\frac{1-P_{e, \text { emp }}}{W_{e}} \quad 0 \leq k \leq W_{e}-1 \\ P\{k \mid k\}=P_{b} \quad 0 \leq k \leq W_{e}-1 \\ P\left\{I_{e, \text { idle }} \mid 0\right\}=P_{e, \text { emp }} \\ P\left\{I_{e, \text { idle }} \mid I_{e, \text { idle }}\right\}=P_{e, \text { emp }} \\ P\left\{k \mid I_{e, \text { idle }}\right\}=\frac{1-P_{e, e m p}}{W_{e}} \quad 0 \leq k \leq W_{e}-1\end{cases}
$$

The meaning of each line in equation (1) is as follows:

1) If the channel is idle, the backoff timer subtracts one;

2) If the backoff timer value is zero and the queue is not empty, then the backoff timer is initially uniformly chosen in $\left[0, W_{e}-1\right]$;

3 ) If the channel is sensed busy, the backoff timer is frozen;

4) If the backoff timer value is zero and the queue is empty, the node is under idle state;

5) If no packets to be sent in the queue, then the node is in the idle state;

6 ) If the queue is empty, a new arriving packet makes the backoff timer uniformly chosen in $\left[0, W_{e}-1\right]$;

The process $\{s(t), b(t)\}$ of WSA traffic is modeled as a two-dimensional Markov chain with discrete-time evolved from [31][33][34], as shown in Fig. 4. Let $s(t)$ and $b(t)$ be a stochastic process standing for the backoff stage and backoff timer of WSA 


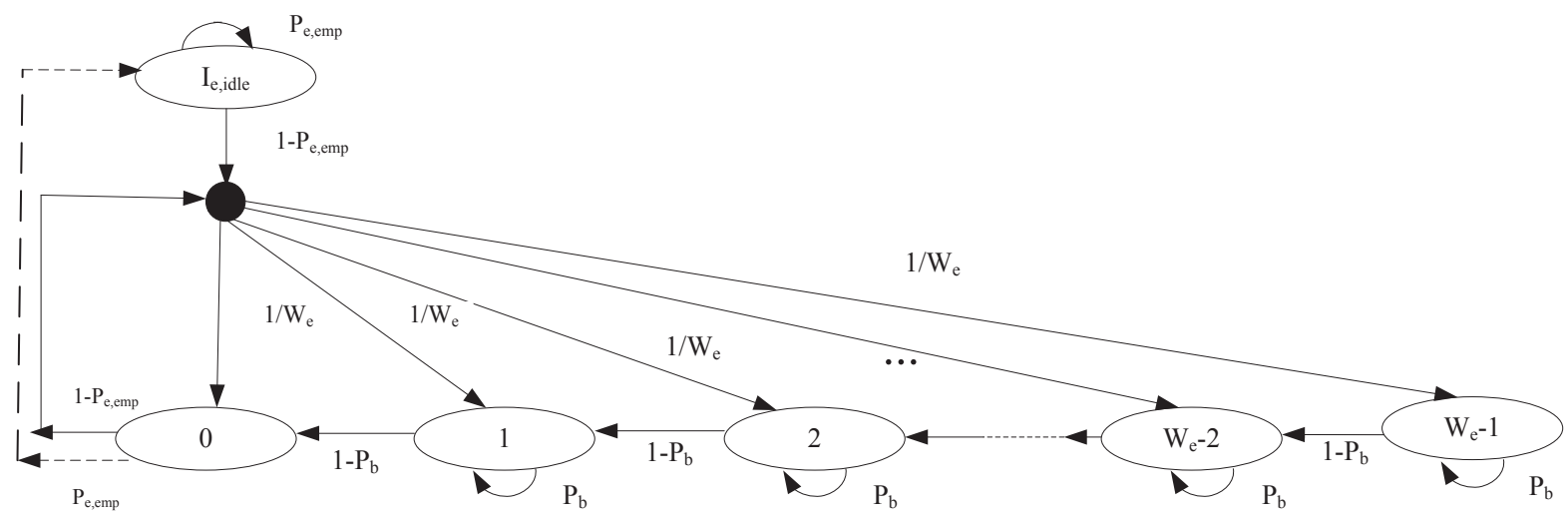

Figure 3: Markov chain model for safety traffic

traffic, respectively. Let the state $I_{s, i d l e}$ represent that the WSA queue of an APDM node is empty. The maximum retransmission number and the $\mathrm{CW}$ in the $i$ th backeoff stage are denoted by $m$ and $W_{s, i}(i \in[0, m])$, respectively. CW is set to the minimum value $W_{s, 0}$ for the first transmission attempt. When the collision is detected, the $\mathrm{CW}$ is doubled and then retransmission is started for the first $m^{\prime}$ steps. While the $\mathrm{CW}$ remains unchanged for $\left(m-m^{\prime}\right)$ steps. To sum up, if $W_{s, i}$ is the CW size in the $i$ th step:

$$
W_{s, i}=\left\{\begin{array}{cc}
2^{i} W_{s, 0} & i \leq m^{\prime} \\
2^{m^{\prime}} W_{s, 0} & m^{\prime}<i \leq m
\end{array}\right.
$$

Let $b_{s, i, k}=\lim _{t \rightarrow \infty}\{s(t)=i, b(t)=k\}, 0 \leq i \leq m, 0 \leq k \leq W_{s, i}-1$ be the stationary distribution of the chain of WSA traffic in Fig. 4. The stationary distribution of the idle state and the backoff state are denoted by $b_{I_{s}}$ and $b_{s, i, k}$, respectively. The 
one-step transition probabilities are:

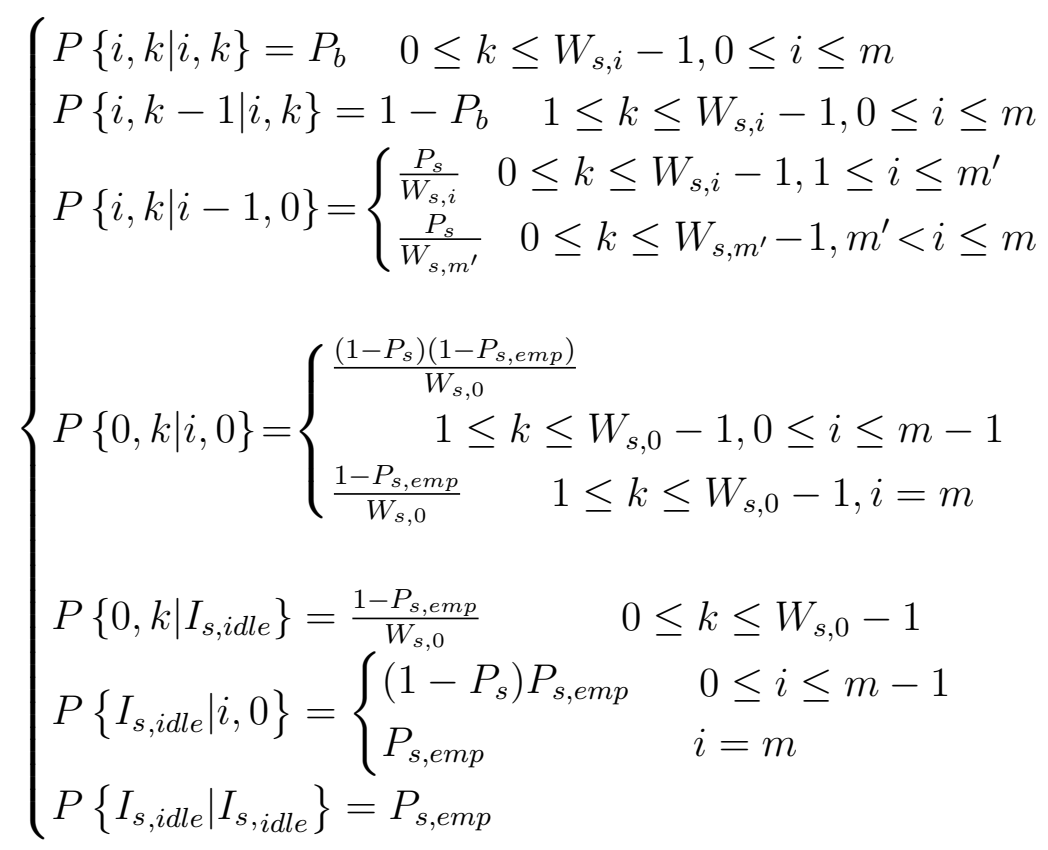

The meaning of each line in equation (3) is as follows:

1) If the channel is busy, the backoff timer is frozen;

2) If the channel is free, the backoff timer will subtract one;

3) Within m' backoff stage, collision makes backoff stage increase and CW double. Otherwise, collision makes CW remain $2^{m} W_{s, 0}$;

4) If a WSA packet is successfully transmitted or a WSA packet reaches its maximum retransmission number $m$, node resets backoff stage;

5) If an APDM node is in state $I_{s, i d l e}$, a new arrived WSA packet makes it enter backoff stage 0 , and then node uniformly chooses backoff timer value in $\left[0, W_{s, 0}-1\right]$;

6) After every successful transmission of the WSA packets, an APDM node enters to state $I_{s, i d l e}$ with a probability of $P_{s, e m p}$;

7) Without a new WSA packet arriving, an APDM node's idle state $I_{s, \text { idle }}$ remains unchanged;

Note that if $P_{e, e m p}=1$ or $P_{s, e m p}=1$, it means that there is no packet to transmit and it is the extreme unsaturated condition. On the other hand, if $P_{e, e m p}=0$ or $P_{s, e m p}=0$, it implies that there is always packet to transmit and it is the saturated condition. Thus, our model covers the extreme unsaturated, intermediate unsaturated, and saturated conditions by taking the queuing status $\left(P_{e, e m p}, P_{s, e m p}\right)$ into account. 




Figure 4: Markov chain model for WSA traffic

\subsection{Safety and WSA Packet Transmission Probability}

Theorem 1. The stationary probability $\tau_{e}$ and $\tau_{s}$ that a node transmits a safety packet and a WSA packet in a generic time slot is $\tau_{e}=b_{e, 0}=\left(\frac{W_{e}-1}{2\left(1-P_{b}\right)}+\frac{1}{1-P_{e, e m p}}\right)^{-1}$ and $\tau_{s}=\frac{1-P_{s}{ }^{m+1}}{1-P_{s}} b_{s, 0,0}$, respectively.

Proof. From the Markov chain in the Fig. 3, we can derive that

$$
\begin{gathered}
b_{I_{e}}=P_{e, e m p} b_{e, 0}+P_{e, e m p} b_{I_{e}} \\
b_{e, k}=\frac{W_{e}-k}{W_{e}\left(1-P_{b}\right)}\left(b_{e, 0} \cdot\left(1-P_{e, e m p}\right)+b_{I_{e}} \cdot\left(1-P_{e, e m p}\right)\right), \\
1 \leq k \leq W_{e}-1
\end{gathered}
$$


Therefore, by imposing the normalization condition, then

$$
b_{I_{e}}+\sum_{k=0}^{W_{e}-1} b_{e, k}=1
$$

Using (4), (5) and (6), variable $\tau_{e}$ can be solved as

$$
\tau_{e}=b_{e, 0}=\left(\frac{W_{e}-1}{2\left(1-P_{b}\right)}+\frac{1}{1-P_{e, e m p}}\right)^{-1}
$$

From the Markov chain in the Fig. 4, we can derive that

$$
b_{s, i-1,0} P_{s}=b_{s, i, 0} \rightarrow b_{s, i, 0}=P_{s}^{i} b_{s, 0,0} \quad 0 \leq i \leq m
$$

Since the Markov chain is regular,we have

$$
\begin{aligned}
b_{s, i, k} & =\frac{W_{s, i}-k}{W_{s, i}\left(1-P_{b}\right)} b_{s, i, 0} \\
& = \begin{cases}\frac{2^{i} W_{s, 0}-k}{2^{i} W_{s, 0}\left(1-P_{b}\right)} b_{s, i, 0}, & 0 \leq i \leq m^{\prime} \\
\frac{2^{m} W_{s, 0}-k}{2^{m} \cdot W_{s, 0}\left(1-P_{b}\right)} b_{s, i, 0}, & m^{\prime}<i \leq m\end{cases}
\end{aligned}
$$

$b_{I_{s}}$ can be derived from

$$
\left(1-P_{s}\right) P_{s, e m p} \sum_{i=0}^{m-1} b_{i, 0}+P_{s, e m p} b_{m, 0}+b_{I_{s}} P_{s, e m p}=b_{I_{s}}
$$

To satisfy the normalization condition for stationary distribution, then :

$$
b_{I_{s}}+\sum_{i=0}^{m} \sum_{k=0}^{W_{s, i}-1} b_{i, k}=1
$$

Combining formula (8), (9), (10) and (11), $b_{s, 0,0}$ can be obtained:

$$
\frac{1}{b_{s, 0,0}}= \begin{cases}\frac{1}{2\left(1-P_{b}\right)}\left(\frac{2 P_{s}-\left(2 P_{s}\right)^{\mathrm{m}+1}}{1-2 P_{s}} W_{s, 0}-\frac{P_{s}-P_{s}^{m+1}}{1-P_{s}}\right) \\ +\frac{1-P_{s}^{m+1}}{1-P_{s}}+\frac{P_{s, e m p}}{1-P_{s, e m p}}, & \left(m \leq m^{\prime}\right) \\ \frac{1}{2\left(1-P_{b}\right)} \frac{2 P_{s}-\left(2 P_{s}\right)^{m}+1}{1-2 P_{s}} W_{s, 0}- & \left.\frac{P_{s}-P_{s}^{m^{\prime}+1}}{1-P_{s}}\right) \\ +\frac{\left(2^{m} W_{s, 0}-1\right)\left(P_{s}^{m^{\prime}+1}-P_{s}^{m+1}\right)}{2\left(1-P_{b}\right)\left(1-P_{s}\right)} & \\ +\frac{1-P_{s}^{m+1}}{1-P_{s}}+\frac{P_{s, e m p}}{1-P_{s, e m p}}, & \left(m>m^{\prime}\right)\end{cases}
$$


Transmission probability $\tau_{s}$ of a WSA packet in a randomly chosen slot time is:

$$
\tau_{s}=\sum_{i=0}^{m} b_{s, i, 0}=\sum_{i=0}^{m} P_{s}^{i} b_{s, 0,0}=\frac{1-P_{s}^{m+1}}{1-P_{s}} b_{s, 0,0}
$$

where $b_{s, 0,0}$ is shown in $(12)$

From (7) and (13), we can see that $\tau_{e}$ and $\tau_{s}$ are expressed solely in terms of $P_{b}$, $W_{e}, P_{e, e m p}$ and $P_{s}, P_{s, e m p}, P_{b}, W_{s, 0}, m^{\prime}, m$, respectively. Place the APDM node in saturation by taking the limit $P_{e, e m p} \rightarrow 0$ and $P_{s, e m p} \rightarrow 0$. In order to determine $P_{e}$, $P_{s}$ and $P_{b}$, we must first determine the relation between the APDM nodes competing for $\mathrm{CCH}$. We do this as follows:

When one more node transmits during a slot time will incur collisions.

$$
\left\{\begin{array}{l}
P_{e}=1-\left(1-\tau_{e}\right)^{N-1}\left(1-\tau_{s}\right)^{N} \\
P_{s}=1-\left(1-\tau_{e}\right)^{N}\left(1-\tau_{s}\right)^{N-1}
\end{array}\right.
$$

The probability $P_{b}$ that the channel is busy is:

$$
P_{b}=1-\left(1-\tau_{e}\right)^{N}\left(1-\tau_{s}\right)^{N}
$$

From equations (7), (13), (14) and (15), we can solve the unknown $\tau_{e}$ and $\tau_{s}$. Note, the expression $\tau_{e}$ includes unknown $P_{e, e m p}$, and the expression $\tau_{s}$ includes unknown $P_{s, e m p}$. Their meanings are shown in Table 2 and their values can be obtained using (19), which will be given in the below.

\subsection{Time Analysis for Safety and WSA Transmission}

In our analytical model, each APDM node's MAC layer has two queues: safety queue and WSA queue to store safety messages and WSA messages, respectively. We assume that the generated safety packets and WSA packets arrive at the MAC layer following a Poisson process with rate $\lambda_{e}$ and $\lambda_{s}$, respectively. According to the APDM MAC and IEEE 1609.4 protocol, only in the $\mathrm{CCH}$ interval, safety and WSA packets can be transmitted on the $\mathrm{CCH}$. If these packets are generated in $\mathrm{SCH}$ interval, they have to wait in the MAC queue until the next $\mathrm{CCH}$ interval to be sent. Therefore, in the APDM MAC protocol, during the CCHI, the arrival rate of safety and WSA packets are the Poisson process with rate $\frac{\beta+1}{\beta} \lambda_{e}$ and $\frac{\beta+1}{\beta} \lambda_{s}$, respectively, where $\beta$ is the ratio between CCHI and SCHI based on the latest SI. The initial duration of CCHI is $50 \mathrm{~ms}(\beta=1)$ and this value is dynamic adjusted according to the traffic condition in the upcoming continuous SI.

Let $P_{e, s u c}$ and $P_{s, s u c}$ be the probability of successful dissemination of a safety packet and a WSA packet, respectively. Let $P_{e, c o l}, P_{s, c o l}$ and $P_{e s, c o l}$ denote the 
probabilities of collision dissemination from only safety packet, only WSA packet and both, respectively. They can be calculated as follows:

$$
\left\{\begin{array}{l}
P_{e, s u c}=N \tau_{e}\left(1-\tau_{e}\right)^{N-1}\left(1-\tau_{s}\right)^{N} \\
P_{s, s u c}=N \tau_{s}\left(1-\tau_{e}\right)^{N}\left(1-\tau_{s}\right)^{N-1} \\
P_{e, c o l}=\left(1-\tau_{s}\right)^{N}\left(1-\left(1-\tau_{e}\right)^{N}-N \tau_{e}\left(1-\tau_{e}\right)^{N-1}\right) \\
P_{s, c o l}=\left(1-\tau_{e}\right)^{N}\left(1-\left(1-\tau_{s}\right)^{N}-N \tau_{s}\left(1-\tau_{s}\right)^{N-1}\right) \\
P_{e s, c o l}=P_{b}-P_{e, s u c}-P_{s, s u c}-P_{e, c o l}-P_{s, c o l}
\end{array}\right.
$$

The transmission of a safety packet and a WSA packet adopts broadcast and unicast way, respectively. According to [35], we have

$$
\left\{\begin{array}{l}
T_{e, s u c}=T_{e, c o l}=S A F E+\delta+D I F S \\
T_{s, s u c}=W S A+A C K+2 \delta+S I F S+D I F S \\
T_{s, c o l}=W S A+\delta+D I F S
\end{array}\right.
$$

Each state may be a state of the channel being idle, successful transmission or a collision. The expected time spent per state $\overline{T_{E S}}$ is

$$
\begin{aligned}
\overline{T_{E S}}= & \left(1-P_{b}\right) \sigma+P_{e, s u c} T_{e, s u c}+P_{s, \text { suc }} T_{s, \text { suc }}+P_{e, c o l} T_{e, c o l} \\
& +P_{s, c o l} T_{s, c o l}+P_{e s, c o l} \cdot \max \left(T_{e, c o l}, T_{s, c o l}\right)
\end{aligned}
$$

During the CCHI, if safety packets and WSA packets arrive at MAC in a Poisson way with rate $\frac{\beta+1}{\beta} \lambda_{e}$ and $\frac{\beta+1}{\beta} \lambda_{s}$, respectively, and then in an expected slot time $\overline{T_{E S}}$, the estimate of $1-P_{e, e m p}$ and $1-P_{s, e m p}$ can be obtained as follows:

$$
\left\{\begin{array}{l}
1-P_{e, e m p}=1-e^{-\frac{\beta+1}{\beta} \lambda_{e} \overline{T_{E S}}} \\
1-P_{s, e m p}=1-e^{-\frac{\beta+1}{\beta} \lambda_{s} \overline{T_{E S}}}
\end{array}\right.
$$

From equations (7), (13), (14), (15), (16), (17), (18) and (19), we can solve the unknowns $\tau_{e}$ and $\tau_{s}$.

Theorem 2. Under unsaturated traffic load conditions, the average of time interval $X_{e}$ and $X_{s}$ are

$$
E\left[X_{e}\right]=\frac{W_{e}-1}{2} \cdot \overline{T_{E S}}+T_{e, s u c}
$$






Proof. When transmitting a safety packet, the node takes the average slot number $\frac{W_{e}-1}{2}$ to perform the backoff. The mean total server time $E\left[X_{e}\right]$ contains the average backoff interval $\frac{W_{e}-1}{2} \overline{T_{E S}}$ and the transmission time $T_{e, s u c}$, as described in (20).

When transmitting a WSA packet, let $Y_{i}$ denote the random variable time that the node spends in $i$ th bachoff stage. The statistical mean of $Y_{i}$ that can be calculated as:

$$
E\left[Y_{i}\right]=\frac{W_{s, i}-1}{2} \cdot \overline{T_{E S}}
$$

Furthermore $i$ is a random variable follows geometric distribution. The average total service time $E\left[X_{s}\right]$ is:

$$
\begin{aligned}
E\left[X_{s}\right] & =\sum_{i=0}^{m} E\left[Y_{i}\right] \cdot P_{s}^{i-1} \cdot\left(1-P_{s}\right)+T_{s, s u c} \\
& =\sum_{i=1}^{m}\left(\frac{W_{s, i}-1}{2} \cdot \overline{T_{E S}}\right) \cdot P_{s}{ }^{i-1} \cdot\left(1-P_{s}\right)+T_{s, s u c}
\end{aligned}
$$

and thus we can achieve the final expression as shown in (21)

\subsection{Optimal Ratio of CCHI and SCHI}

According to Fig. 2, we have

$$
\left\{\begin{array}{l}
T_{\text {cch }}=T_{\text {safe }}^{\prime}+T_{w s a}^{\prime} \\
T_{\text {total }}=T_{\text {cch }}+T_{\text {sch }}
\end{array}\right.
$$

Let $G_{1}$ be the number of WSA reservation made on $\mathrm{CCH}$ during the random contention interval.

$$
T_{w s a}^{\prime}=G_{1} \cdot E\left[X_{s}\right]
$$

The ratio $\beta$ between $T_{c c h}$ and $T_{\text {sch }}$ can be expressed by

$$
\beta=\frac{T_{c c h}}{T_{s c h}}
$$


To ensure the timely and reliable delivery of safety packets, we must set aside enough time for all safety-related packets transmission.

$$
T_{\text {safe }}^{\prime}=N\left(1-e^{-\frac{\beta+1}{\beta} \lambda_{e} \overline{T_{E S}}}\right) E\left[X_{e}\right]
$$

For simplicity, we assume the service packet length is constant, the time to transmit a service packet on $\mathrm{SCH}$ is calculated by

$$
T_{\text {data }}=T_{h}+D A T A+S I F S+A C K+D I F S+2 \delta
$$

where $T_{h}$ is the cost of MAC header and PHY header attached to the service packet. $D A T A=L / V_{\text {sch }}$, and $V_{\text {sch }}$ and $L$ represent the data transmission rate on SCHs and the payload of a service packet, respectively.

Let $G_{2}$ denote the number of service packets delivered on $N_{\text {sch }}$ SCHs during the SCHI. We have

$$
T_{\text {sch }}=\frac{G_{2} T_{\text {data }}}{N_{\text {sch }}}
$$

The optimum ratio between CCHI and SCHI only when $G_{1}=G_{2}$, that is the number of reservations made on $\mathrm{CCH}$ is equal to the number of service packets disseminated on $N_{\text {sch }}$ SCHs. For vehicle nodes, in the CCHI, no enough idle time slots left to make more reservations. On the other hand, in the SCHI, no enough idle time slots left to transmit more service packets [9]. From (24), (25), (26), (27), (28) and (29) we can update the value $\beta$ based on (30).

$$
\begin{aligned}
\beta & =\frac{T_{c c h}}{T_{\text {sch }}} \\
= & \left.\frac{\frac{T_{\text {data }}}{N_{\text {sch }}} \cdot N \cdot\left(1-e^{-\frac{\beta+1}{\beta} \lambda_{e} \overline{T_{E S}}}\right) \cdot E\left[X_{e}\right]+T_{\text {total }} \cdot E\left[X_{s}\right]}{\frac{T_{\text {data }}^{\prime}}{N_{\text {sch }}} \cdot\left(T_{\text {total }}-N \cdot\left(1-e^{-\frac{\beta+1}{\beta} \lambda_{e} \bar{T}_{E S}}\right) \cdot E\left[X_{e}\right]\right.}\right) \\
= & \frac{N\left(1-e^{-\frac{\beta+1}{\beta} \lambda_{e} \overline{T_{E S}}}\right) E\left[X_{e}\right]+T_{\text {total }} E\left[X_{s} \frac{N_{\text {sch }}}{T_{\text {data }}}\right.}{\left(T_{\text {total }}-N\left(1-e^{-\frac{\beta+1}{\beta} \lambda_{e} \bar{T}_{E S}}\right) E\left[X_{e}\right]\right)}
\end{aligned}
$$

\subsection{Performance Analysis}

Let $E\left[D_{e}^{1609.4}\right]$ and $E\left[D_{e}^{A P D M}\right]$ denote the delay of transmitting a safety packet in IEEE 1609.4 protocol and APDM MAC protocol, respectively. For simplicity, each node is modeled as two independent $\mathrm{M} / \mathrm{M} / 1$ queues and the buffer size of queues is infinite for both safety traffic and WSA traffic. The average service time of a safety 
packet and a WSA packet is denoted by $\frac{1}{\mu_{e}}$ and $\frac{1}{\mu_{s}}$, respectively. It can be seen that $\frac{1}{\mu_{e}}=E\left[X_{e}\right]$ and $\frac{1}{\mu_{s}}=E\left[X_{s}\right][36]$.

In IEEE 1609.4 protocol, before being disseminated, the safety packets already have the delay of $T_{s c h i} / 2$, and the average delay of the safety packets is

$$
E\left[D_{e}^{1609.4}\right]=\frac{T_{s c h i}}{2}+\frac{1}{\mu_{e}^{*}-2 \lambda_{e}}=\frac{T_{\text {total }}}{4}+\frac{1}{\mu_{e}^{*}-2 \lambda_{e}}
$$

where $\frac{1}{\mu_{e}^{*}}=E\left[X_{e}^{*}\right]$ means that $E\left[X_{e}^{*}\right]$ is equal to $E\left[X_{e}\right]$, when $\beta$ is constant and its value is 1 in $E\left[X_{e}\right]$.

For the APDM MAC protocol, a safety packet is already by delaying $T_{\text {sch }} / 2$ before it is arranged to arrive at MAC queue to deliver, and the average delay until the safety packet is successfully transmitted is calculated as

$$
E\left[D_{e}^{A P D M}\right]=\frac{T_{s c h}}{2}+\frac{1}{\mu_{e}-\frac{\beta+1}{\beta} \lambda_{e}}
$$

In APDM MAC protocol, the total delay to transmit a service packet includes two parts: the delay in the CCHI $D_{c c h}$ and the delay in the SCHI $D_{\text {sch }}$, which can be expressed by (33) and (34), respectively. Therefore, we can easily derive the average transmission delay of a service packet.

$$
\begin{gathered}
E\left[D_{c c h}\right]=\frac{1}{2}\left(G_{1}+1\right) \cdot E\left[X_{s}\right] \\
E\left[D_{s c h}\right]=\frac{\left[\frac{1}{2}\left\lfloor\frac{G_{1}}{N_{s c h}}\right\rfloor \cdot N_{s c h}+\left(G_{1} \bmod N_{s c h}\right)\right]}{G_{1}} \\
\cdot\left[\left\lfloor\frac{G_{1}}{N_{s c h}}\right\rfloor+1\right] \cdot E\left[T_{\text {data }}\right]
\end{gathered}
$$

where the symbol $\lfloor\cdot\rfloor$ is a floor function.

The aggregate throughput of the service traffic of the proposed APDM MAC protocol is expressed by

$$
S^{A P D M}=\frac{T_{s c h}}{E\left[T_{d a t a}\right]} \cdot N_{s c h} \cdot L
$$

\section{Performance Evaluation}

We have carried out extensive simulations to verify our proposed APDM MAC protocol. The simulation scenario is a $2-\mathrm{km}$ of a 4 -lane highway as shown in Fig. 5 . 
The average vehicles speed is $60 \mathrm{~km} / \mathrm{h}$, and every vehicle has a GPS and a single-radio WAVE communication device. It is assumed that there is the same transmission data rate on the $\mathrm{CCH}$ and SCHs. All nodes can act as both service providers and service users, and are under the transmission range of each. The generated safety packets and WSA packets arrive at the MAC layer in Poisson distributions. Therefore, the network density can be increased either by increasing the number of vehicles while fixing the Poisson arriving rate of safety packets and WSA packets or by increasing the Poisson arriving rate of safety packets and WSA packets while fixing the number of vehicles. Our simulations cover the different saturated levels of queues: extreme unsaturated, intermediate unsaturated and saturated conditions by changing Poisson arrival rate. On the other hand, according to the requirement in communicationenabled vehicular safety-related applications, the minimum transmission rate and maximum transmission rate are $1 \mathrm{~Hz}$ and $50 \mathrm{~Hz}$ [19], respectively. Therefore, the packet arrival rate with 1pps (packets per second) and 50pps can be used to represent extreme non-saturated case and intermediate non-saturated case in our simulations, respectively. In general, packet arrival rate with 1000pps, the queue can be ensured with saturated traffic load [17][18], and thus, this value can be used to simulate saturated conditions. For facilitation of the analysis, we set the value of $\lambda_{e}$ equal the value of $\lambda_{s}$. We conduct simulation experiments by using NS-3 [37]. All configuration parameters are listed in the table 3 .

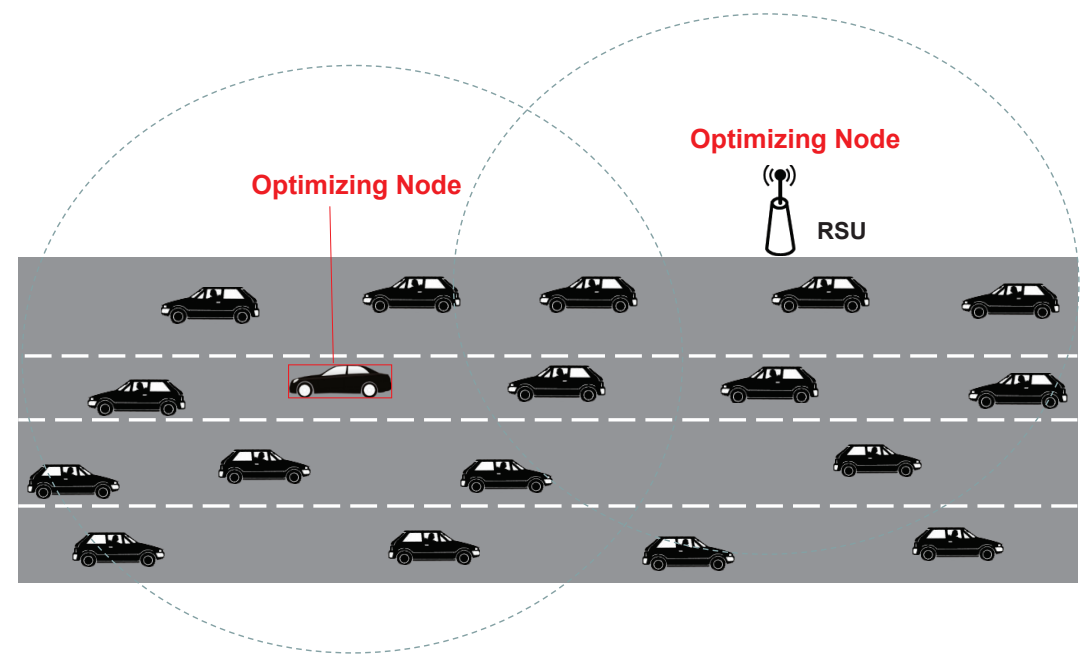

Figure 5: 4-lane highway scenario with optimizing nodes (ONs) and moving vehicles

The transmission probability of the safety-related messages versus the $\mathrm{CCH}$ inter- 
Table 3: SYSTEM PARAMETER FOR SIMULATIONS

\begin{tabular}{cc}
\hline Parameters & Values \\
\hline Number of SCHs & 4 \\
Number of CCH & 1 \\
Date rate for each channel & $3 \mathrm{Mbps}$ \\
$W_{e}$ & 8 \\
$W_{s, 0}$, & 32 \\
$m^{\prime}$ & 5 \\
$\mathrm{~m}$ & 10 \\
MAC header & $256 \mathrm{bits}$ \\
PHY header & $192 \mathrm{bits}$ \\
WSA & PHY header $+160 \mathrm{bits}$ \\
ACK & PHY header $+112 \mathrm{bits}$ \\
$T_{s i f s}$ & $10 \mu \mathrm{s}$ \\
$T_{d i f s}$ & $50 \mu \mathrm{s}$ \\
$\sigma$ & $20 \mu \mathrm{s}$ \\
$\delta$ & $1 \mu \mathrm{s}$ \\
Variable packet arrival rate & $\lambda_{e}=\lambda_{s}$ \\
The length of service packet & $2000 \mathrm{bytes}$ \\
Number of nodes & 30 \\
\hline
\end{tabular}


val is given in Fig. 6. ${ }^{1}$ We can see that, with higher $\mathrm{CCH}$ interval, the transmission of the safety-related packets is guaranteed. The higher of packet arrival rate, the more $\mathrm{CCH}$ interval is required. When the duration of the $\mathrm{CCH}$ interval is $33.6 \mathrm{~ms}, 97.9 \mathrm{~ms}$ and $128 \mathrm{~ms}$, for packet arrival rate with 1 pps, 50pps and $1000 \mathrm{pps}$, all safety-related packets can be successfully disseminated. The analytical results and simulation results match well each other, our proposed model is validated.

The throughput on SCHs in terms of the CCHI is shown in Fig. 7. It can be observed that, according to the level of saturation, the curves show different variations. When Poisson arrival rates of packets is 1 pps or 50pps, the throughput increases first then decreases. This is because when the CCH interval is less than $33.6 \mathrm{~ms}$ (packets arrival with 1pps) or $97.9 \mathrm{~ms}$ (packets arrival with 50pps), the nodes has only little chance to make reservations. On the contrary, when the $\mathrm{CCH}$ interval is larger, due to the shortage of the $\mathrm{SCH}$ interval, too many reservations on $\mathrm{CCH}$ interval are infeasible. When Poisson arrival rate is $1000 \mathrm{pps}$, the $\mathrm{CCH}$ interval of $100 \mathrm{~ms}$ is all used to transmit the safety messages, WSA has no chance to make reservation, thus, the throughput is zero. The analytical results are matched well simulation results. Thus the proposed APDM MAC protocol can be validated. However, the throughput achieved by simulation is slightly less than that achieved by analysis. The reason is that, in the simulation experiment, the time is wasted if the remaining time on $\mathrm{SCH}$ interval is insufficient for transmitting a service packet. While in the analytical model, all service packets have adequate time to be disseminated since it is assumed that both CCHI and SCHI are infinite,

In order to investigate the optimal ratio of transmission probabilities with different priorities, the analytical result is demonstrated in Fig. 8. As can be observed in the figure, when the Poisson arrival rates for both safety and WSA packet are low (e.g, $\lambda_{\mathrm{e}}=\lambda_{\mathrm{s}}=1 \mathrm{pps}$ ), and thus the nodes are in extreme unsaturated conditions, the channel competition is not severe, the ratio changes little. However, with the Poisson arrival rate of packets increasing, the ratio significantly becomes larger with the growth of $N$. If $N$ is fixed, the ratio is also larger as the Poisson arrival rate gets higher. For example, when $N$ is 60 , the Poisson arrival rates for safety packets and WSA packets are $\lambda_{\mathrm{e}}=\lambda_{s}=1000 \mathrm{pps}$, the ratio is 12.70 , while the ratio is 2.03 when $\lambda_{\mathrm{e}}=\lambda_{s}=50$ pps. This is because with the increasement of the number of nodes or especially the Poisson arrival rate growing, more collisions will occur on

\footnotetext{
${ }^{1}$ Note: the legend in figure, in each row, if there are two number, the first number and the second number represent $\lambda_{\mathrm{e}}$ and $\lambda_{\mathrm{s}}$, respectively. If there is only one number in each row, the number denotes $f_{V C I}$ for VCI protocol, where $f_{V C I}$ (packets/second) denotes the sending frequent of safety packets. The following figures is the same.
} 


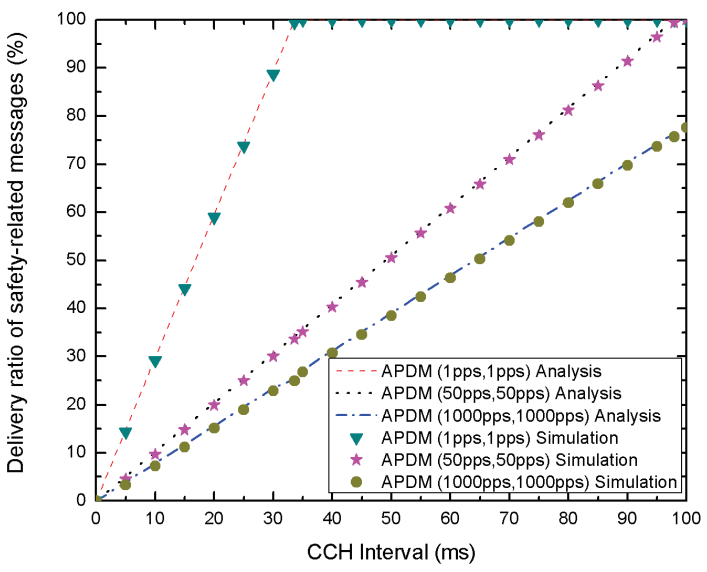

Figure 6: Ensure the transmission of safety-related messages

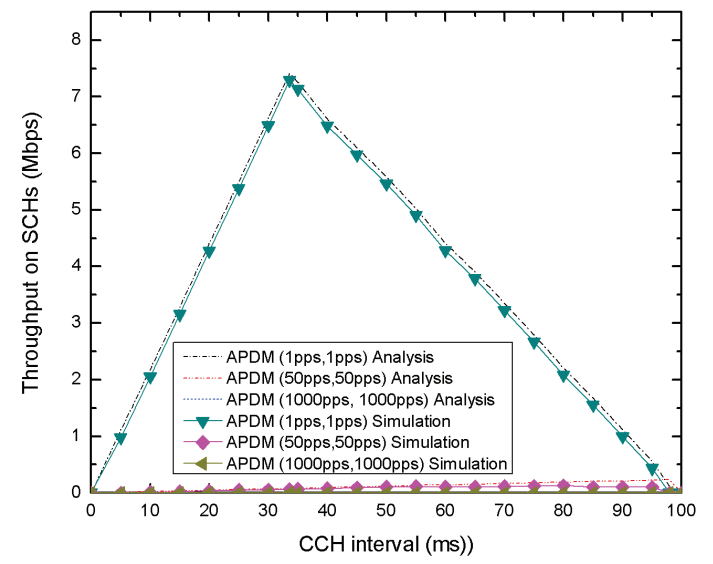

Figure 7: Throughput on SCHs versus $\mathrm{CCH}$ interval

the $\mathrm{CCH}$, and the transmission probability of the WSA packets will decrease due to more backoff nodes.

We compare our proposed APDM MAC protocol with original IEEE 1609.4 protocol [4] and VCI protocol [9].

Fig. 9 shows the optimal $\mathrm{CCH}$ interval under various conditions using the different protocols. Fig. 9(a) demonstrates the optimal $\mathrm{CCH}$ interval versus the number of nodes. The $\mathrm{CCH}$ interval increases when either $N$ increases or the Poisson arrival rates of packets (safety packets or WSA packets) grows. This is because when $N$ or $\lambda_{\mathrm{e}}, \lambda_{\mathrm{s}}$ increases, the probability of channel collisions increases, and thus the network is required to set aside more time to safety message. From the result, we can conclude that by providing longer $\mathrm{CCH}$ interval in dense circumstances, our proposed APDM $\mathrm{MAC}$ protocol can guarantee reliable transmission of safety packets and at the same 


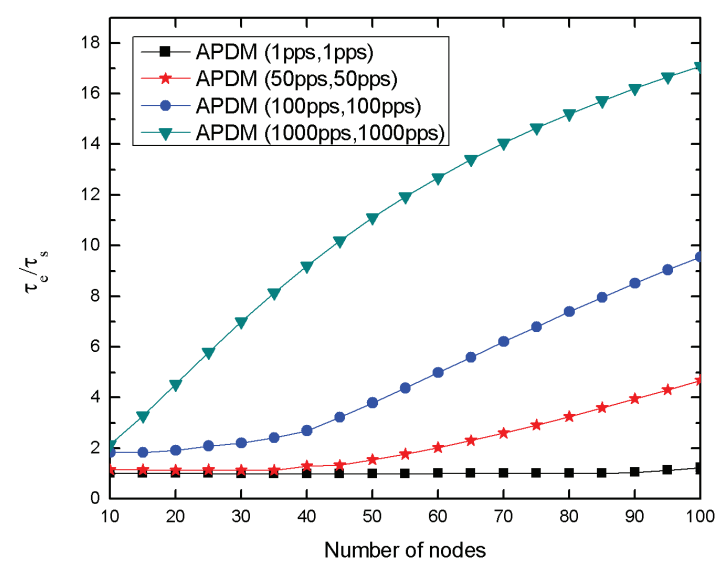

Figure 8: Optimal ratio of transmission probabilities

time, by performing more non-safety application in light traffic load, it can improve the channel utilization. When the network traffic is heavy, such as $\lambda_{\mathrm{e}}=\lambda_{\mathrm{s}}=f_{V C I}=$ 50 pps, with the number of nodes increasing, the VCI protocol quickly reaches $100 \mathrm{~ms}$ $(N=6)$, and APDM MAC protocol steady rises to $100 \mathrm{~ms}(N=55)$. On the other hand, in a light network traffic, especially under conditions of less safety packets, WSA packets and number of nodes, the optimal $\mathrm{CCH}$ interval given by APDM is much smaller than the CCH interval given by VCI. For example, when $N=5$, the optimal $\mathrm{CCH}$ interval of $\mathrm{APDM}\left(\lambda_{\mathrm{e}}=\lambda_{\mathrm{s}}=1 \mathrm{pps}\right)$ is $5.4 \mathrm{~ms}$ and $\mathrm{SCH}$ interval is $94.6 \mathrm{~ms}$, while the CCH interval of VCI $\left(f_{V C I}=1 \mathrm{pps}\right)$ is $30.3 \mathrm{~ms}$ and SCH interval is $69.7 \mathrm{~ms}$. This is because the VCI protocol is under saturated conditions when every node always has safety and WSA packets available to transmit and APDM MAC protocol is under unsaturated conditions that each node has s probability that the buffer is empty. Moreover, the APDM MAC protocol takes burst characteristic of emergency into consideration which is a more important characteristic of VANETs. Fig. 9(b) shows the optimal CCH interval versus the service packet length. As illustrated, the optimal CCH interval decreases. It can be seen that APDM and VCI protocol are both able to adjust the CCHI and SCHI to provide suitable bandwidth according to different traffic load. Fig. 9(c) gives the optimal $\mathrm{CCH}$ interval in terms of the Poisson arrival rate of safety and WSA packets for APDM , the sending frequency of safety packets for VCI. As can be seen in the figure, with the rising of $\lambda_{e}, \lambda_{s}$ or $f_{V C I}$, the $\mathrm{CCH}$ interval increases. This is an expected behavior because when $\lambda_{e}, \lambda_{s}$ or $f_{V C I}$ raises, the systems will set aside enough time to ensure reliable dissemination of safety packets. When the arrival rates of packets for APDM MAC and VCI protocol are same, the $\mathrm{CCH}$ interval in APDM MAC protocol is less than the $\mathrm{CCH}$ interval in VCI protocol, and thus, APDM MAC protocol can leave more SCH interval to 
service packet transmissions. For example, when packet arrival rate is 10pps, APDM MAC protocol sets aside $56.4 \mathrm{~ms}$ to $\mathrm{CCH}$ interval and $43.6 \mathrm{~ms}$ to $\mathrm{SCH}$ interval. While VCI protocol leaves $100 \mathrm{~ms}$ to $\mathrm{CCH}$ interval and zero time to $\mathrm{SCH}$ interval, and this does not qualify the characters of vehicular networks. The reason is that in VANETs, the transmission rate for the safety message such as beacon message is usually 10pps [13][14]. When $N$ is 30, the vehicular network is under light or moderate traffic load, it can leaves time for $\mathrm{SCH}$ interval without compromising the transmissions of safety messages.

From Fig. 9(a), 9(b) and 9(c), we can conclude that APDM and VCI MAC protocol can adjust the CCHI according to $N$, service packet length and the arrival rate $\left(\lambda_{e}, \lambda_{s}\right.$ in APDM, $f_{V C I}$ in $\left.\mathrm{VCI}\right)$ of the packets. However, the original IEEE 1609.4 protocol has constant intervals of $\mathrm{CCH}$ and $\mathrm{SCH}$ under various conditions, and it lacks adaptive features. Compared to VCI protocol, the APDM MAC protocol can ensure the timely and reliable transmission of safety message, meanwhile, it can leave more time to $\mathrm{SCH}$ interval.

The effect of number of nodes on SCHs throughput is investigated in Fig. 10. The service packet length is 2000 bytes. As the figure shows, curves have decreasing trend as $N$ increases in APDM MAC and VCI protocol. This is because the collision probability increases with $N$ increasing. Therefore, these two protocols set aside more time to CCHI and give SCHI less time. In light network traffic $\left(\left(\lambda_{e}=\lambda_{s}=\right.\right.$ 1pps for both IEEE 1609.4 and APDM, $f_{V C I}=1 \mathrm{pps}$ for VCI), compared to the IEEE 1609.4 and VCI protocol, APDM MAC protocol improves the throughput on SCHs by $132 \%$ and $11 \%$, respectively. The reason is that APDM leaves more time to SCHs interval than that of the VCI and IEEE 1609.4, which can be seen from Fig. 9 (a). On the other hand, under dense network traffic environments (e.g, $\lambda_{e}=\lambda_{s}=$ 50 pps or $1000 \mathrm{pps}, f_{V C I}=50 \mathrm{pps}$ or $1000 \mathrm{pps}$ ), the throughput in APDM is still higher than that of VCI and IEEE 1609.4 protocol. Since the APDM can leave more time to SCHs interval than VCI does. Although the IEEE 1609.4 have more time $(50 \mathrm{~ms})$ for SCHs interval than the APDM does, which can be seen from Fig. 9(a), the nodes using APDM MAC protocol can transmit service packets without contention.

The system throughput on SCHs versus the length of service packets with 30 nodes is given in Fig. 11. Again, in our proposed APDM MAC protocol, the analytical results match with the simulation results very well. It can be observed that the APDM MAC protocol greatly outperforms the original IEEE 1609.4 protocol in light traffic load. When $\lambda_{e}=\lambda_{s}=1 \mathrm{pps}$ and the service packet length is 2000 bytes, the APDM MAC protocol can bring about approximately $60 \%$ improvement from original IEEE 1609.4. This is because when in light traffic load network, the proposed APDM MAC protocol leaves more time to SCHs as shown in Fig. 9(b), 




(a)

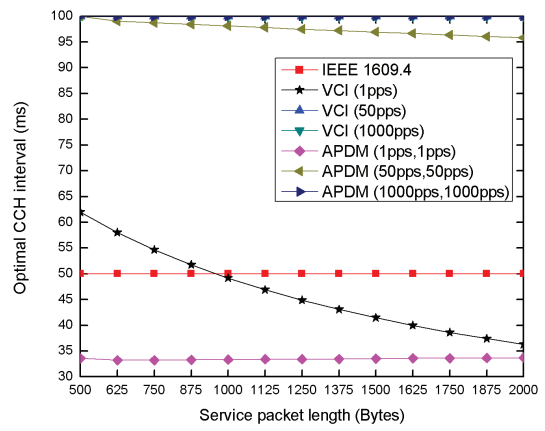

(b)

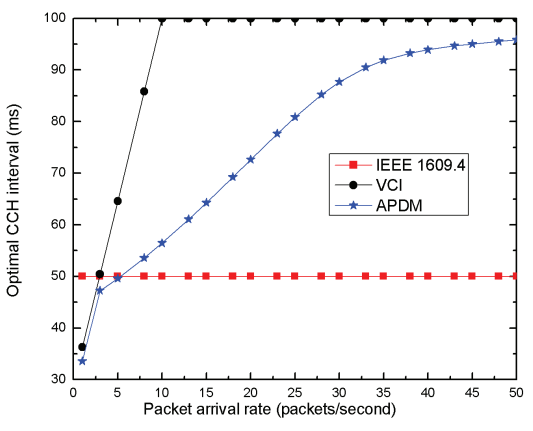

(c)

Figure 9: Optimal CCH interval. (a) In terms of the number of nodes. (b) In terms of the service packet length. (c) In terms of the Poisson arrival rate of safety packets $\lambda_{e}=\lambda_{s}$ for APDM and the sending frequency of safety packets $f_{V C I}$ for VCI. 
and the nodes adopting the APDM MAC protocol have capability of sending service packets with contentions-free. When $\lambda_{e}=\lambda_{s}=50 \mathrm{pps}$ and the length of service packet is 2000 bytes, compared with IEEE 1609.4 protocol, the APDM MAC protocol improves the throughput on SCHs by $323 \%$. In extreme non-saturated conditions $\left(\lambda_{e}=\lambda_{s}=f_{V C I}=1 \mathrm{pps}\right)$ and the length of service packet is 500 bytes, the throughput on SCHs of APDM improves by $74 \%$ than that of VCI. While in intermediate nonsaturated conditions $\left(\left(\lambda_{e}=\lambda_{s}=f_{V C I}=50 \mathrm{pps}\right)\right)$, the throughput of VCI protocol is zero. And thus, whenever in extreme unsaturated condition or in intermediate unsaturated condition, the performance in the APDM MAC protocol outperforms the VCI MAC protocol. This is because the APDM MAC protocol adjusts more time to SCHs, which can be seen from Fig. 9(b).

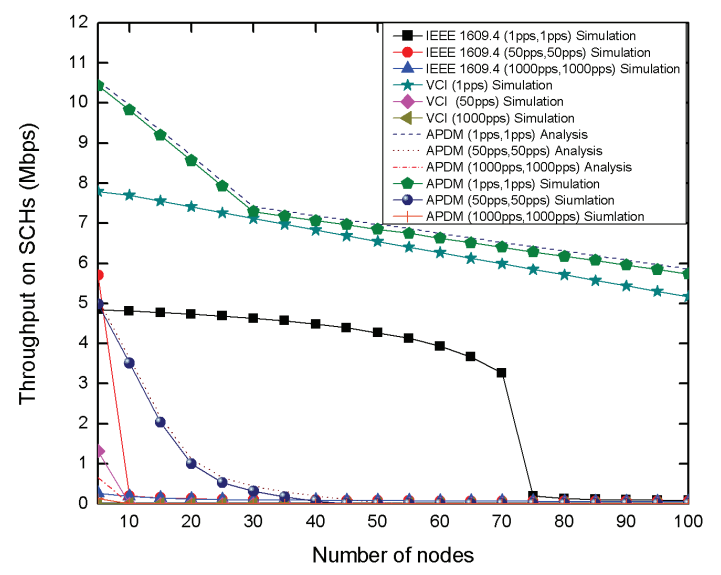

Figure 10: Throughput results versus the number of nodes

Fig. 12 shows the throughput on SCHs versus the Poisson arrival rate of safety and WSA packets for APDM and IEEE 1609.4 protocol, the sending frequency $f_{V C I}$ of safety packets for VCI protocol. The curves have decreasing trend as packet arrival rate increases. For APDM and VCI protocol, since the SCH interval decreases as the packet arrival rate increases. Nevertheless, in IEEE 1609.4 protocol, the collision probability of service packets increase as the Poisson arrival rate of packets increases. $\lambda_{e}$ or $f_{V C I}$. When $\lambda_{e}=\lambda_{s}=1 \mathrm{pps}$ and $\lambda_{e}=\lambda_{s}=50 \mathrm{pps}$, the average throughput on SCHs for APDM MAC protocol is $60 \%$ and $320 \%$, respectively, which is higher than that using the original IEEE 1609.4 protocol. However, there is a considerable difference between APDM and VCI. The throughput flattens down with the rise of Poisson arrival rate $\left(\lambda_{e}=\lambda_{s}\right)$ in APDM, while the throughput decreases rapidly with the rise of the $f_{V C I}$ in VCI. The reason is that, in APDM MAC protocol, we assume that the nodes providing the services are under unsaturated conditions, i.e., every 


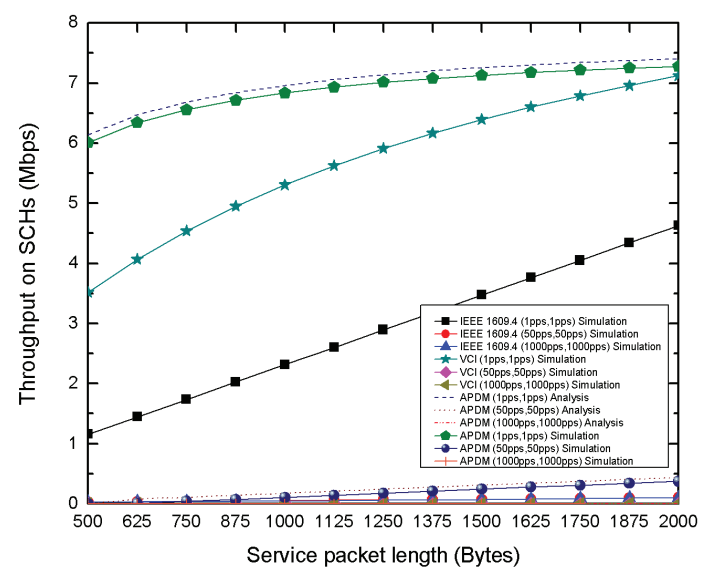

Figure 11: Throughput results versus the service packet length

node generating packets arrive at the MAC layer in a Poisson way. However, the VCI MAC protocol is under saturated environments, which means every node has packets available in MAC layer to transmit. On the premise of reliable transmission of safe packets, APDM MAC protocol can leave more time for SCH interval, as can be seen from Fig. 9(c).

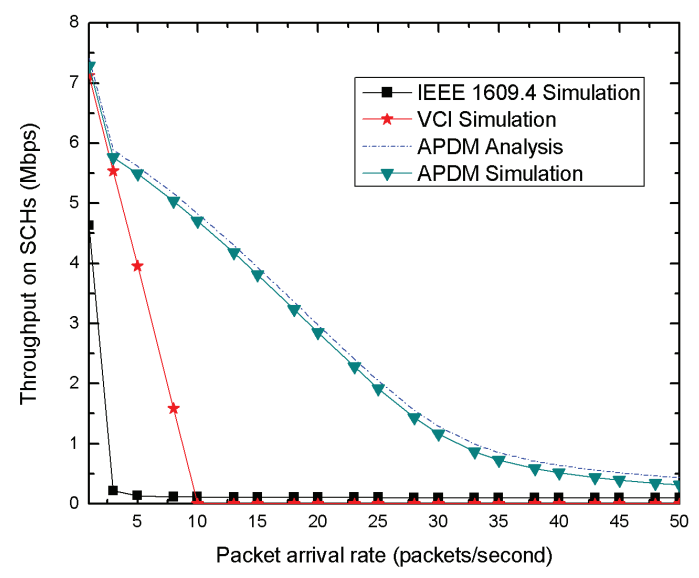

Figure 12: Throughput results versus the Poisson arrival rate of safety packets $\lambda_{e}=\lambda_{s}$ for APDM and the sending frequency of safety packets $f_{V C I}$ for VCI.

The effect of number of nodes on average safety packets delay is illustrated in Fig. 13. The average safety packets delay is defined as the sum of time that safety packets spend in SCHI and CCHI. According to the APDM and IEEE 1609.4 protocol, during 
SCHI, the safety packets arriving at MAC layer will wait until the next beginning of SI to transmit, and thus the safety packets need to wait for average half of the SCHI. As the figure shows, the safety packets delay has increasing trend as $N$ increases in IEEE 1609.4. This is because the average service time $E\left[X_{e}^{*}\right]=\frac{1}{\mu_{e}^{*}}$ of the safety packets becomes longer as a result of more fierce competition due to $N$ increasing. Nevertheless, we find that when nodes are in saturated conditions, such as $\left(\lambda_{e}=\lambda_{s}\right)$ $=1000 \mathrm{pps}$, the delay is less than the nodes are in extreme unsaturated condition $\left(\lambda_{e}\right.$ $\left.=\lambda_{s}\right)=1 \mathrm{pps}$ ) and intermediate unsaturated condition $\left.\left(\lambda_{e}=\lambda_{s}\right)=50 \mathrm{pps}\right)$, since in the saturated condition, the WSA packets have no chance to be transmitted. The delay of safety packet in APDM MAC protocol consists of two parts, as can be seen from (31). According to the APDM MAC protocol, the larger number of nodes requires longer $\mathrm{CCH}$ interval. Therefore, the average delay of safety packets declines with the increasing number of nodes. In heavy traffic condition such as $\lambda_{e}=\lambda_{s}=$ 50 pps, the delay performance of safety packets using APDM MAC protocol improves by $422 \%$ than that using the IEEE 1609.4 .

Fig. 14 shows the delay of safety packets against Poisson arrival rate of packets. It can be seen that the APDM MAC protocol can ensure reliable and timely transmission of safety-related packets according to this figure. As $\lambda_{e}$ and $\lambda_{s}$ grows, the safety packet delay of APDM MAC protocol rapidly decreases and the average delay of IEEE 1609.4 increases. Since the APDM MAC protocol increases CCHI as packet arrival rate increases, and it decrease SCHI to ensure the safety packets timely transmission. In APDM MAC protocol, when $\lambda_{e}=\lambda_{s}=50 \mathrm{pps}$, the safety packet delay performance improves by $495 \%$ than that in IEEE 1609.4, respectively.

Fig. 15 shows the average service packet delay versus the service packet length. The delay of service packet increases with the payload of packet increasing, since the long packet need more time to transmit. The APDM outperforms the IEEE 1609.4 according to the service packet delay. This is because, unlike the IEEE 1609.4, the APDM can offer the chance for a more contention-free transmission for service packets. When $\lambda_{e}=\lambda_{s}=1 \mathrm{pps}$ and $\lambda_{e}=\lambda_{s}=50 \mathrm{pps}$, the packet size is 2000 bytes. The delay performance of APDM MAC protocol improves by $35 \%$ and $218 \%$ than that of IEEE 1609.4 protocol. 


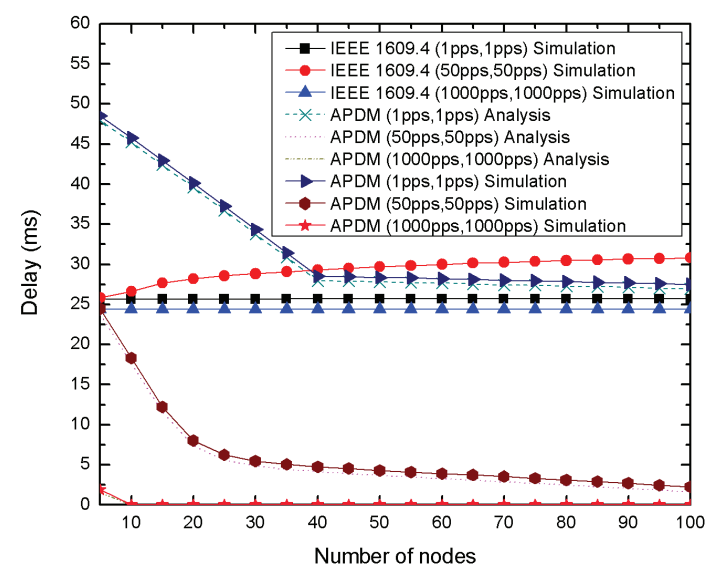

Figure 13: Average safety packets delay versus the number of nodes

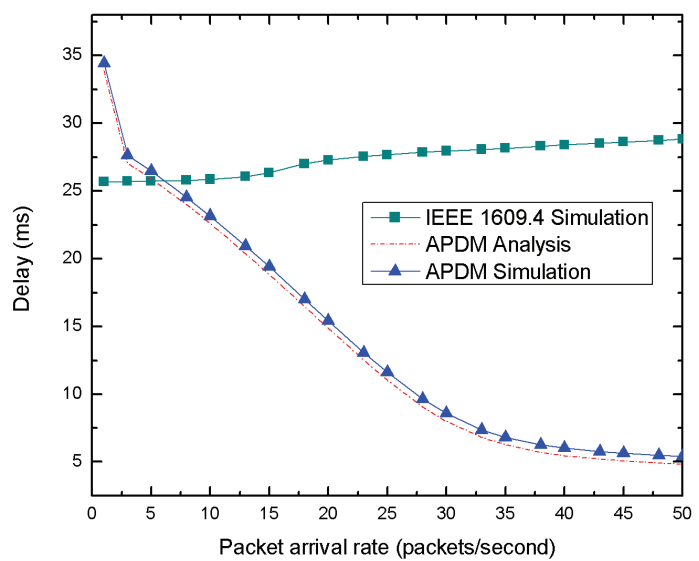

Figure 14: Average safety packet delay in terms of $\lambda_{e}$ 




Figure 15: Average service packet delay under different packet lengths 


\section{CONCLUSION AND FUTURE WORKS}

This paper proposes an adaptive multi-priority multichannel distributed (APDM) MAC protocol under unsaturated conditions. The APDM MAC protocol can reduce the safety packet delay on $\mathrm{CCH}$ and improve the system throughput on SCHs in VANETs. Therefore, it can satisfy both the delay-sensitive and throughput-sensitive applications. By giving different priorities to different classes of information, the safety-related packets with higher priorities can be preferentially sent on $\mathrm{CCH}$ under different traffic environments. We assume that the generated packets arrive at MAC layer follow a Poisson process. A Markov chain analytical model is then used to derive the optimal $\mathrm{CCH}$ interval, and the $\mathrm{M} / \mathrm{M} / 1$ queue model is adopted to analyze the time performance. The analysis and simulation are under unsaturated conditions to reflect the characteristic of safety packets burst in real VANETs scenarios. Both analytical and simulation results manifest that the APDM MAC protocol can ensure reliable and timely transmission of safety packets, and improve the channel utilization. Our future work is to research how to reduce safety competition on $\mathrm{CCH}$ by power and rate control.

\section{Acknowledgments}

This work was supported in part by the Foundation of National 863 Plan of China under Grant 2012AA111902, in part by the National Natural Science Foundation of China No. 61471084, in part by National Science Foundation of China under Grant 61502072, in part by the Fundamental Research Funds for the Central Universities No. DUT15QY02, in part by the Fundamental Research Funds for the Central Universities of China under Grant DUT14RC(3)064, and in part by the Post-Doctoral Science Foundation of China under Grant 2014M561229 and 2015T80251

\section{References}

[1] G. Karagiannis, O. Altintas, E. Ekici, G. Heijenk, B. Jarupan, K. Lin, T. Weil, Vehicular networking: A survey and tutorial on requirements, architectures, challenges, standards and solutions, Communications Surveys Tutorials, IEEE 13 (4) (2011) 584-616. doi:10.1109/SURV.2011.061411.00019.

[2] H. T. Cheng, H. Shan, W. Zhuang, Infotainment and road safety service support in vehicular networking: From a communication perspective, Mechanical Systems and Signal Processing 25 (6) (2011) 2020 - 2038, interdisciplinary Aspects of Vehicle Dynamics. doi:http://dx.doi.org/10.1016/j.ymssp.2010.11.009. 
[3] W. G. Spadafora, P. M. Paielli, D. R. Llewellyn, J. G. Kramer, Intelligent transportation system, uS Patent 7,689,230 (Mar. 30 2010).

[4] IEEE standard for wireless access in vehicular environments (WAVE)-multichannel operation, IEEE Std 1609.4-2010 (Revision of IEEE Std 1609.4-2006) (2011) 1-89doi:10.1109/IEEESTD. 2011.5712769.

[5] H. Yoo, D. Kim, Dynamic channel coordination schemes for ieee 802.11 p/1609 vehicular networks: A survey, International Journal of Distributed Sensor Networks 2013.

[6] C. Campolo, A. Molinaro, A. Vinel, Y. Zhang, Modeling prioritized broadcasting in multichannel vehicular networks, Vehicular Technology, IEEE Transactions on 61 (2) (2012) 687-701. doi:10.1109/TVT.2011.2181440.

[7] C. Campolo, A. Molinaro, Multichannel communications in vehicular ad hoc networks: a survey, Communications Magazine, IEEE 51 (5) (2013) 158-169. doi:10.1109/MCOM. 2013.6515061.

[8] L. Liu, W. Xia, L. Shen, An adaptive multi-channel MAC protocol with dynamic interval division in vehicular environment, in: Information Science and Engineering (ICISE), 2009 1st International Conference on, 2009, pp. 2534-2537. doi:10.1109/ICISE. 2009.226.

[9] Q. Wang, S. Leng, H. Fu, Y. Zhang, An ieee 802.11p-based multichannel MAC scheme with channel coordination for vehicular ad hoc networks, Intelligent Transportation Systems, IEEE Transactions on 13 (2) (2012) 449-458. doi:10.1109/TITS.2011.2171951.

[10] C. Shao, S. Leng, Y. Zhang, H. Fu, A multi-priority supported ppersistent MAC protocol for vehicular ad hoc networks (2012) 25322537doi:10.1109/WCNC. 2012.6214225.

[11] L. Zhang, Z. Liu, R. Zou, J. Guo, Y. Liu, A scalable CSMA and self-organizing TDMA MAC for ieee $802.11 \mathrm{p} / 1609 . \mathrm{x}$ in VANETs, Wireless Personal Communications 74 (4) (2014) 1197-1212.

[12] Y. Kim, M. Lee, T. Lee, Coordinated multichannel MAC protocol for vehicular ad hoc networks, Vehicular Technology, IEEE Transactions on PP (99) (2015) 1-1. doi:10.1109/TVT.2015.2475165. 
[13] G. Bansal, J. B. Kenney, C. E. Rohrs, LIMERIC: A linear adaptive message rate algorithm for DSRC congestion control, IEEE Transactions on Vehicular Technology 62 (9) (2013) 4182-4197. doi:10.1109/TVT. 2013. 2275014.

[14] F. Daneshgaran, M. Laddomada, F. Mesiti, M. Mondin, Unsaturated throughput analysis of IEEE 802.11 in presence of non ideal transmission channel and capture effects, IEEE Transactions on Wireless Communications 7 (4) (2008) 1276-1286. doi:10.1109/TWC. 2008.060859.

[15] X. Ma, X. Chen, H. H. Refai, Unsaturated performance of IEEE 802.11 broadcast service in vehicle-to-vehicle networks, in: 2007 IEEE 66th Vehicular Technology Conference, 2007, pp. 1957-1961. doi:10.1109/VETECF.2007.411.

[16] H. Qiu, I.-H. Ho, C. Tse, Y. Xie, A methodology for studying 802.11p VANET broadcasting performance with practical vehicle distribution, Vehicular Technology, IEEE Transactions on 64 (10) (2015) 4756-4769. doi:10.1109/TVT . 2014.2367037.

[17] C. Han, M. Dianati, R. Tafazolli, R. Kernchen, X. Shen, Analytical study of the IEEE 802.11p MAC sublayer in vehicular networks, IEEE Transactions on Intelligent Transportation Systems 13 (2) (2012) 873-886. doi:10.1109/TITS. 2012.2183366.

[18] Y. Yao, L. Rao, X. Liu, Performance and reliability analysis of ieee 802.11p safety communication in a highway environment, IEEE Transactions on Vehicular Technology 62 (9) (2013) 4198-4212. doi:10.1109/TVT. 2013.2284594.

[19] H. Hartenstein, K. Laberteaux, VANET: vehicular applications and internetworking technologies, Vol. 1, Wiley Online Library, 2010.

[20] S.-I. Sou, Modeling emergency messaging for car accident over dichotomized headway model in vehicular ad-hoc networks, Communications, IEEE Transactions on 61 (2) (2013) 802-812. doi:10.1109/TCOMM. 2012.010913.110368.

[21] M. Javed, J. Khan, D. Ngo, Joint space-division multiple access and adaptive rate control for basic safety messages in VANETs, in: Wireless Communications and Networking Conference (WCNC), 2014 IEEE, 2014, pp. 2688-2693. doi:10.1109/WCNC. 2014.6952853. 
[22] C. Han, M. Dianati, R. Tafazolli, X. Liu, X. Shen, A novel distributed asynchronous multichannel MAC scheme for large-scale vehicular ad hoc networks, Vehicular Technology, IEEE Transactions on 61 (7) (2012) 3125-3138. doi:10.1109/TVT.2012.2205596.

[23] D. N. M. Dang, C. S. Hong, S. Lee, E.-N. Huh, An efficient and reliable MAC in VANETs, Communications Letters, IEEE 18 (4) (2014) 616-619. doi:10.1109/LCOMM. 2014.030114.132504.

[24] H. Yoo, J. Kim, D. Kim, A dynamic safety interval protocol for VANETs, in: Proceedings of the 2012 ACM Research in Applied Computation Symposium, ACM, 2012, pp. 209-214. doi:10.1145/2401603.2401650.

[25] K. Hafeez, L. Zhao, B. Ma, J. Mark, Performance analysis and enhancement of the DSRC for VANET's safety applications, Vehicular Technology, IEEE Transactions on 62 (7) (2013) 3069-3083. doi:10.1109/TVT.2013.2251374.

[26] X. Ma, X. Chen, Performance analysis of IEEE 802.11 broadcast scheme in ad hoc wireless LANs, Vehicular Technology, IEEE Transactions on 57 (6) (2008) 3757-3768. doi:10.1109/TVT.2008.918731.

[27] K. Hafeez, L. Zhao, J. Mark, X. Shen, Z. Niu, Distributed multichannel and mobility-aware cluster-based MAC protocol for vehicular ad hoc networks, Vehicular Technology, IEEE Transactions on 62 (8) (2013) 3886-3902. doi:10.1109/TVT.2013.2258361.

[28] H. Su, X. Zhang, Clustering-based multichannel MAC protocols for QoS provisionings over vehicular ad hoc networks, Vehicular Technology, IEEE Transactions on 56 (6) (2007) 3309-3323. doi:10.1109/TVT.2007.907233.

[29] H. Omar, W. Zhuang, L. Li, VeMAC: A TDMA-based MAC protocol for reliable broadcast in VANETs, Mobile Computing, IEEE Transactions on 12 (9) (2013) 1724-1736. doi : 10.1109/TMC. 2012.142.

[30] M. Khabazian, S. Aissa, M. Mehmet-Ali, Performance modeling of message dissemination in vehicular ad hoc networks with priority, Selected Areas in Communications, IEEE Journal on 29 (1) (2011) 61-71. doi:10.1109/JSAC. 2011.110107.

[31] G. Bianchi, Performance analysis of the IEEE 802.11 distributed coordination function, Selected Areas in Communications, IEEE Journal on 18 (3) (2000) 535-547. doi:10.1109/49.840210. 
[32] J. Mao, Y. Mao, S. Leng, X. Bai, Performance optimization for IEEE 802.11 with QoS differentiation supporting, Journal of Software 21 (11) (2010) 28662882 .

[33] E. Ziouva, T. Antonakopoulos, CSMA/CA performance under high traffic conditions: throughput and delay analysis, Computer communications 25 (3) (2002) 313-321. doi:10.1016/S0140-3664(01)00369-3.

[34] M. M. Gharehajlu, Y. Darmani, S. Zokaei, Delay analysis of IEEE 802.11 based ad-hoc network under unsaturated condition, Wireless Personal Communications 79 (2) (2014) 1455-1470. doi:10.1007/s11277-014-1940-7.

[35] IEEE standard for wireless access in vehicular environments (WAVE)- network systems, IEEE Std 1609.3-2010.

[36] D. Malone, K. Duffy, D. Leith, Modeling the 802.11 distributed coordination function in nonsaturated heterogeneous conditions, IEEE/ACM Transactions on Networking 15 (1) (2007) 159-172. doi:10.1109/TNET. 2006.890136.

[37] NS-3, available: https://www.nsnam.org/releases/. 